\title{
Synthesis of Novel pyrimido[4,5-b]quinoline-4-one Derivatives and Assessment as Antimicrobial and Antioxidant Agents
}

\author{
Usama Fathy ${ }^{1, *}$, Rasha S. Gouhar ${ }^{2}$, Ahmed Younis ${ }^{3}$, Dina H. El-Ghonemy ${ }^{4}$
}

Usama Fathy ${ }^{1, *}$, Rasha S. Gouhar ${ }^{2}$, Ahmed Younis ${ }^{3}$, Dina H. ElGhonemy ${ }^{4}$

'Applied Organic Chemistry Department, National Research Centre, 33 El Bohouth st. (former EL Tahrir st.)-Dokki-Giza-P.O.12622, EGYPT.

${ }^{2}$ Therapeutic Chemistry Department, National Research Centre, 33 El Bohouth st. (former EL Tahrir st.)-Dokki-Giza-P.O.12622, EGYPT.

${ }^{3}$ Green Chemistry Department, National Research Centre, 33 El Bohouth st. (former EL Tahrir st.)-Dokki-Giza-P.O.12622, EGYPT. ${ }^{4}$ Microbial Chemistry Department, Genetic Engineering and Biotechnology Division, National Research Centre, 33 El Buhouth St., Dokki, Cairo, P. C. 12622, EGYPT.

\section{Correspondence}

Usama Fathy

Applied Organic Chemistry Department, National Research Centre, 33 El Bohouth

st. (former EL Tahrir st.)-Dokki-Giza-

P.O.12622, EGYPT.

E-mail: usamafathy2000@yahoo.com

History

- Submission Date: 19-10-2020;

- Review completed: 14-12-2020;

- Accepted Date: 30-12-2020.

DOI : 10.5530/pj.2021.13.69

Article Available online http://www.phcogj.com/v13/i2

Copyright

(C) 2021 Phcogj.Com. This is an openaccess article distributed under the terms of the Creative Commons Attribution 4.0 International license.

\section{ABSTRACT}

Objective: Antimicrobial resistance has emerged as one of the serious global health problems of the $21^{\text {st }}$ century that threatens the efficient treatment and prevention of an everincreasing range of infections caused by bacteria, viruses, and fungi. Therefore, it would be favorable to find promising agents with antioxidant and antimicrobial activity combined in one molecule. Key findings: Pyrimido[4,5-b] quinolines are biologically active compounds that are known to rely primarily on the functional group's existence and location. Quinolinbenzo-[1,3]oxazin-4-one (3) was prepared and played as electrophilic interface/mediator for the synthesis of many compounds, such as pyrimido[4,5-b]quinoline, quinoline-carboxamide and oxoquinazolin-acetamide by reacting with nucleophilic reagent. Summary: Results revealed that pyrimido[4,5-b] quinoline derivatives (17b, 9d and $\mathbf{9 c}$ ) are the most potent compounds that displayed significant antimicrobial activity along with compounds $17 a, 29 b, 5,19,23 b$, and $25 \mathrm{~b}$ that appeared to be more promising as antioxidant agents than ascorbic acid.

Key words: Quinoline, Benzoxazinones, Pyrimidoquinolin, Antimicrobial agent, Antioxidant agent.

\section{INTRODUCTION}

The compounds based on scaffold of quinolines have been reported to possess a wide range of pharmaceutical properties ${ }^{1-7}$. Several structures based on quinoline have proved effective inhibitors of important proteins from microbial pathogens ${ }^{8}$. The modified classes of compounds based on quinolines have been studied recently for their antimicrobial $^{9,10}$. Quinoline-carboxamide I, II, III were reported as the most potent EGFR inhibitors with $\mathrm{IC}_{50} 2.6,0.49$ and $1.73 \mathrm{mM}$, respectively ${ }^{11}$. Iminosugar/Azasugars fused benzo $[1,3]$ thiazin4-one exhibited significant HIV-RT inhibitory activities $^{12,13}$ (Figure 1).

Pyrimido[4,5-b]quinolin-4-ones were reported as analgesic, anti-inflammatory, and antimicrobial ${ }^{14}$ antimitotic agents and cytotoxic activity ${ }^{15}$. Pyrrolidine-2,5-dione showed antioxidant, antidiabetic activity ${ }^{16}$ analgesic and antiallodynic activity $^{17}$.

Benzoxazinones which are widely used in pharmaceutics have a wide range of pharmaceutical activities for example, ntiphlogistic,, antifungal, antibacterial ${ }^{18}$, anti-human coronavirus ${ }^{19}$, inhibitor of human leucocyte elastase, anti-cathepsin $\mathrm{G}$, complement protein receptor blocker ${ }^{20}$ and chymotrypsin antagonist ${ }^{21}$. Benzo[1,3] oxazin-4ones (IV) showed high significant against DNA-PK, PI3K,PDE3A enzymes and platelet aggregation ${ }^{22}$. Benzoxazinones IV have showed antioxidant and anticancer activity ${ }^{20}$ (Figure 1).

Based on that, we decided to complete the work on pyrimido[4,5-b]quinoline and synthesis of benzo[d][1,3] oxazin-4-one as stating material for new compounds and evaluating their antioxidant and antimicrobial activity.

\section{MATERIALS AND METHODS}

\section{Equipments}

All melting points are uncorrected and were taken on open capillary tubes using electrothermal apparatus 9100. Elemental micro analyses were carried out at microanalytical unit, Central Services Laboratory, National Research Centre, Dokki, Cairo-Egypt, using Vario Elementar and were found within + or $-0.5 \%$ of the theoretical values. Infrared spectra were recorded on a Jasco FT/IR-6100, Fourier Transform Infrared Spectrometer at $\mathrm{cm}^{-1}$ scale using $\mathrm{KBr}$ disc technique at the Central Services Lab. NRC, Dokki, Cairo, Egypt. ${ }^{1} \mathrm{HNMR}$ spectra were determined by using a JEOL EX-270 NMR Spectrometer at Central Services Lab, NRC. Mass spectra were measured with Finnigan M A T SSQ-7000 mass spectrometer at the Central Services, NRC Dokki, Cairo, Egypt. Follow up of the reactions and checking the purity of the compounds were made by TLC on silica gelprecoated aluminum sheets (Type 60 F254-Merck, Darmstadt, Germany) and the spots were detected by exposure to UV Lamp at 254 nanometer for few seconds.

\section{Chemistry synthesis}

Synthesis of 2-(10-cyclohexyl-5-(3,4dimethoxyphenyl)-4-oxo-3,4,5,6,7,8,9,10-octahydropyrimido[4,5-b]quinolin-2-yl)-4H-benzo[d][1,3] oxazin-4-one (3)

To a solution of anthranilic acid (1.371 g, 0.01 mole) in dry pyridine $(30 \mathrm{~mL}), 10$-cyclohexyl-5(3,4-dimethoxyphenyl)-4-oxo-3,4,5,6,7,8,9,10- 




octahydropyrimido[4,5-b]quinoline-2-carbonyl chloride (1) $(0.02$ mole) was added portion wise with stirring at room temperature for $12 \mathrm{hrs}$. The reaction mixture was poured onto cold water $(100 \mathrm{~mL})$ and the precipitated solid was filtered off, washed with cold water, dried and recrystallized from ethanol to give benzo[d][1,3]xazin-4-one derivative 3.

Yellow crystals; Yield $60 \%$; m.p. $123-124{ }^{\circ} \mathrm{C}$; IR $\left(\mathrm{KBr}, \mathrm{cm}^{-1}\right): 3450$ $(\mathrm{NH}), 1745(\mathrm{C}=\mathrm{O}), 1715(\mathrm{C}=\mathrm{O}), 1654,1680(\mathrm{C}=\mathrm{N}), 1243$ (aryl ethers); ${ }^{1} \mathrm{H}-\mathrm{NMR}\left(500 \mathrm{MHz}, \mathrm{DMSO}-\mathrm{d}_{6}, \delta / \mathrm{ppm}\right): 1.45\left(m, 2 \mathrm{H}, \mathrm{CH}_{2}\right), 1.50-1.81$ $\left(m, 4 \mathrm{H}, 2 \mathrm{CH}_{2}\right), 1.52\left(m, 2 \mathrm{H}, \mathrm{CH}_{2}\right), 1.65\left(m, 2 \mathrm{H}, \mathrm{CH}_{2}\right), 1.74(m, 2 \mathrm{H}$, $\left.\mathrm{CH}_{2}\right), 1.75-2.20\left(q, 4 \mathrm{H}, 2 \mathrm{CH}_{2}\right), 1.82\left(m, 2 \mathrm{H}, \mathrm{CH}_{2}\right), 2.50(m, 1 \mathrm{H}, \mathrm{CH})$, $3.72\left(s, 3 \mathrm{H}, \mathrm{OCH}_{3}\right), 3.80\left(s, 3 \mathrm{H}, \mathrm{OCH}_{3}\right), 4.40(s, 1 \mathrm{H}, \mathrm{CH}), 6.61-6.85$ ( $m, 3 \mathrm{H}, \operatorname{Ar}-\mathrm{H}), 7.21-8.42(d, 4 \mathrm{H}, \mathrm{Ar}-\mathrm{H}), 10.50(s, 1 \mathrm{H}, \mathrm{NH}) ; \mathrm{MS}(\mathrm{m} / z$, (relative abundance, \%)): $566\left(\mathrm{M}^{+}, 30\right)$; Anal. Calcd. for $\mathrm{C}_{33} \mathrm{H}_{34} \mathrm{~N}_{4} \mathrm{O}_{5}: \mathrm{C}$, 69.95; H, 6.05; N, 9.89; Found: C, 69.98; H, 6.02; N, 9.90.

Synthesis of $\mathrm{N}-(2-((2-a m i n o p h e n y l)$ carbamoyl)phenyl)-10-cyclohexyl-5(3,4-dimethoxyphenyl)-4-oxo-3,4,5,6,7,8,9,10-octahydropyrimido[4,5b]quinoline-2-carboxamide (5)

A mixture of benzoxazinone $2(1.18 \mathrm{~g}, \quad 0.003$ mole $)$ and $o$-phenylenediamine $(0.32 \mathrm{gm}, 0.003 \mathrm{~mole})$ in $\mathrm{EtOH}(20 \mathrm{~mL})$ was refluxed for 8 hours. The solid product that separated on cooling was filtered off and recrystallized from ethanol to give $\mathbf{5}$.

Reddish brown crystals; Yield 40\%; m.p. 101-103 ${ }^{\circ} \mathrm{C}$; IR $\left(\mathrm{KBr}, \mathrm{cm}^{-1}\right)$ : $3521\left(\mathrm{NH}_{2}\right), 3450,3425,3390(\mathrm{NH}), 1750,1740,1720(\mathrm{C}=\mathrm{O}), 1690$, $1665(\mathrm{C}=\mathrm{N}), 1265(\mathrm{OMe}) ;{ }^{1} \mathrm{H}-\mathrm{NMR}\left(500 \mathrm{MHz}, \mathrm{DMSO}-\mathrm{d}_{6}, \delta / \mathrm{ppm}\right)$ : $1.45\left(m, 2 \mathrm{H}, \mathrm{CH}_{2}\right), 1.50-1.81\left(m, 4 \mathrm{H}, 2 \mathrm{CH}_{2}\right), 1.52\left(t, 2 \mathrm{H}, \mathrm{CH}_{2}\right), 1.65$ $\left(m, 2 \mathrm{H}, \mathrm{CH}_{2}\right), 1.74\left(m, 2 \mathrm{H}, \mathrm{CH}_{2}\right), 1.75-2.20\left(q, 4 \mathrm{H}, 2 \mathrm{CH}_{2}\right), 1.82(t, 2 \mathrm{H}$, $\left.\mathrm{CH}_{2}\right), 2.50(m, 1 \mathrm{H}, \mathrm{CH}), 3.78\left(s, 3 \mathrm{H}, \mathrm{OCH}_{3}\right), 3.80\left(s, 3 \mathrm{H}, \mathrm{OCH}_{3}\right), 4.20$ $(s, 1 \mathrm{H}, \mathrm{NH}), 4.40(s, 1 \mathrm{H}, \mathrm{CH}), 5.50\left(s, 1 \mathrm{H}, \mathrm{NH}_{2}\right), 6.61-7.89(m, 11 \mathrm{H}, \mathrm{Ar}-$ $\mathrm{H}), 9.30(s, 1 \mathrm{H}, \mathrm{NH}), 10.10(s, 1 \mathrm{H}, \mathrm{NH}), 11.05(s, 1 \mathrm{H}, \mathrm{NH})$; MS $(\mathrm{m} / z$, (relative abundance, \%)): $674\left(\mathrm{M}^{+}, 35\right)$; Anal. Calcd. for $\mathrm{C}_{39} \mathrm{H}_{42} \mathrm{~N}_{6} \mathrm{O}_{5}: \mathrm{C}$, 69.42; H, 6.27; N, 12.45; Found: C, 69.20; H, 5.79; N, 12.28 .

Synthesis of 10-cyclohexyl-5-(3,4-dimethoxyphenyl)-2-(4-oxo-3,4- dihydroquinazolin-2-yl)-5, 6, 7, 8,9, 10-hexahydropyrimido[4,5-b] quinolin-4(3H)-one (6)

A mixture of benzoxazinone 3 (3.93 g, 0.01 mole) and $\mathrm{CH}_{3} \mathrm{COONH}_{4}$ $(2.68 \mathrm{~g}, 0.01 \mathrm{~mole})$ was fused in an oil bath. The reaction mixture was left to cool, washed with water several times, filtered off, dried and recrystallized from ethanol to give 6.

Yellow crystals; Yield 45\%; m.p. 114- $115^{\circ} \mathrm{C}$; IR $\left(\mathrm{KBr}, \mathrm{cm}^{-1}\right): 3440,3337$ $(\mathrm{NH}), 1729,1715(\mathrm{C}=\mathrm{O}), 1637,1624(\mathrm{C}=\mathrm{N}), 1290(\mathrm{OMe}) ;{ }^{1} \mathrm{H}-\mathrm{NMR}$ (500 MHz, DMSO-d $\left.\mathrm{d}_{6}, \delta / \mathrm{ppm}\right): 1.45\left(m, 2 \mathrm{H}, \mathrm{CH}_{2}\right), 1.50-1.81(m, 4 \mathrm{H}$, $\left.2 \mathrm{CH}_{2}\right), 1.52\left(t, 2 \mathrm{H}, \mathrm{CH}_{2}\right), 1.65\left(m, 2 \mathrm{H}, \mathrm{CH}_{2}\right), 1.74\left(m, 2 \mathrm{H}, \mathrm{CH}_{2}\right), 1.75-$ $2.20\left(q, 4 \mathrm{H}, 2 \mathrm{CH}_{2}\right), 1.82\left(t, 2 \mathrm{H}, \mathrm{CH}_{2}\right), 2.50(m, 1 \mathrm{H}, \mathrm{CH}), 3.78(s, 3 \mathrm{H}$, $\left.\mathrm{OCH}_{3}\right), 3.81\left(s, 3 \mathrm{H}, \mathrm{OCH}_{3}\right), 4.40(s, 1 \mathrm{H}, \mathrm{CH}), 6.61-7.90(m, 7 \mathrm{H}, \mathrm{Ar}-\mathrm{H})$, $11.10(s, 1 \mathrm{H}, \mathrm{NH}), 11.20(\mathrm{~s}, 1 \mathrm{H}, \mathrm{NH}) ; \mathrm{MS}(\mathrm{m} / \mathrm{z}$, (relative abundance, \%)): $565\left(\mathrm{M}^{+}, 55\right)$; Anal. Calcd. for $\mathrm{C}_{33} \mathrm{H}_{35} \mathrm{~N}_{5} \mathrm{O}_{4}: \mathrm{C}, 70.07 ; \mathrm{H}, 6.24 ; \mathrm{N}$, 12.38; Found: C, 69.79; H, 6.60; N, 12.02 .

Synthesis of 10-cyclohexyl-5-(3,4-dimethoxyphenyl)-2-(4-thioxo-4Hbenzo [d] [1,3] thiazin-2-yl)-5,6,7,8,9,10-hexahydropyrimido[4,5-b] quinolin-4(3H)-one (7)

A mixture of benzoxazinone 3 (3.93g, 0.01 mole) and $\mathrm{P}_{2} \mathrm{~S}_{5}(8.9 \mathrm{~g}, 0.02$ mole) in dry xylene $(40 \mathrm{~mL})$ was refluxed for $8 \mathrm{~h}$. The reaction mixture was filtered off while hot, concentrated and the solid that separated on cooling was washed with petroleum ether (b.p. $80-100^{\circ}$ ), then recrystallized from ethanol to give 7.

Yellow crystals; Yield 53\%; m.p. 146-148 ${ }^{\circ} \mathrm{C}$; IR $\left(\mathrm{KBr}, \mathrm{cm}^{-1}\right): 3398(\mathrm{NH})$, $1725(\mathrm{C}=\mathrm{O}), 1646,1633(\mathrm{C}=\mathrm{N}), 1219$ (OMe), 1150 (C=S); ${ }^{1} \mathrm{H}-\mathrm{NMR}$ (500 MHz, DMSO-d $\left.\mathrm{d}_{6}, \delta / \mathrm{ppm}\right): 1.45\left(m, 2 \mathrm{H}, \mathrm{CH}_{2}\right), 1.50-1.81(m, 4 \mathrm{H}$, $\left.2 \mathrm{CH}_{2}\right), 1.52\left(t, 2 \mathrm{H}, \mathrm{CH}_{2}\right), 1.65\left(m, 2 \mathrm{H}, \mathrm{CH}_{2}\right), 1.74\left(m, 2 \mathrm{H}, \mathrm{CH}_{2}\right), 1.75-$ $2.20\left(q, 4 \mathrm{H}, 2 \mathrm{CH}_{2}\right), 1.82\left(t, 2 \mathrm{H}, \mathrm{CH}_{2}\right), 2.50(m, 1 \mathrm{H}, \mathrm{CH}), 3.78(s, 3 \mathrm{H}$, $\left.\mathrm{OCH}_{3}\right), 3.82\left(s, 3 \mathrm{H}, \mathrm{OCH}_{3}\right), 4.40(s, 1 \mathrm{H}, \mathrm{CH}), 6.91-7.30(m, 7 \mathrm{H}, \mathrm{Ar}-\mathrm{H})$, $11.20(s, 1 \mathrm{H}, \mathrm{NH})$; $\mathrm{MS}(\mathrm{m} / z$, (relative abundance, $\%)): 598\left(\mathrm{M}^{+}, 29\right)$; Anal. Calcd. for $\mathrm{C}_{33} \mathrm{H}_{34} \mathrm{~N}_{4} \mathrm{O}_{3} \mathrm{~S}_{2}: \mathrm{C}, 68.19 ; \mathrm{H}, 5.88 ; \mathrm{N}, 9.61 ; \mathrm{S}, 5.5$ Found: C, 66.48; H, 5.23; N, 9.32, S, 4.48. 


\section{Synthesis of compounds $9 a-d$}

A solution of benzoxazinone 2 ( $3.93 \mathrm{~g}, 0.01 \mathrm{~mole})$ and amine derivatives namely, hydrazine hydrate, $p$-aminopyridine, 4-bromoaniline, or 4-aminoacetophenone $(0.02$ mole $)$ in absolute EtOH $(30 \mathrm{~mL})$ was refluxed for 6 hours. The solid product that separated on cooling was filtered off, dried and recrystallized from ethanol to afford the quinazolinone derivative $9 \mathrm{a}-\mathrm{d}$.

2-(3-amino-4-oxo-3,4-dihydroquinazolin-2-yl)-10-cyclohexyl-5-(3,4dimethoxyphenyl) $\quad-5,6,7,8,9,10$-hexahydropyrimido[4,5-b]quinolin4(3H)-one (9a)

Yellow crystals; Yield 63\%; m.p. 109-110 ${ }^{\circ} \mathrm{C}$; IR $\left(\mathrm{KBr}, \mathrm{cm}^{-1}\right): 3500,3437$ (NH), 1744, $1728(\mathrm{C}=\mathrm{O}), 1630,1615(\mathrm{C}=\mathrm{N}), 1220(\mathrm{OMe}) ;{ }^{1} \mathrm{H}-\mathrm{NMR}$ (500 MHz, DMSO-d $\left.\mathrm{d}_{6}, \delta / \mathrm{ppm}\right): 1.45\left(m, 2 \mathrm{H}, \mathrm{CH}_{2}\right), 1.50-1.81(m, 4 \mathrm{H}$, $\left.2 \mathrm{CH}_{2}\right), 1.52\left(t, 2 \mathrm{H}, \mathrm{CH}_{2}\right), 1.65\left(m, 2 \mathrm{H}, \mathrm{CH}_{2}\right), 1.74\left(m, 2 \mathrm{H}, \mathrm{CH}_{2}\right), 1.75-$ $2.20\left(q, 4 \mathrm{H}, 2 \mathrm{CH}_{2}\right), 1.82\left(t, 2 \mathrm{H}, \mathrm{CH}_{2}\right), 2.50(m, 1 \mathrm{H}, \mathrm{CH}), 3.78(s, 3 \mathrm{H}$, $\left.\mathrm{OCH}_{3}\right), 3.80\left(s, 3 \mathrm{H}, \mathrm{OCH}_{3}\right), 4.40(s, 1 \mathrm{H}, \mathrm{CH}), 5.50\left(s, 2 \mathrm{H}, \mathrm{NH}_{2}\right), 6.61-$ $7.70(m, 7 \mathrm{H}, \mathrm{Ar}-\mathrm{H}), 11.30(\mathrm{~s}, 1 \mathrm{H}, \mathrm{NH}) ; \mathrm{MS}(\mathrm{m} / z$, (relative abundance, \%)): $580\left(\mathrm{M}^{+}, 65\right)$; Anal. Calcd. for $\mathrm{C}_{33} \mathrm{H}_{36} \mathrm{~N}_{6} \mathrm{O}_{4}: \mathrm{C}, 68.26 ; \mathrm{H}, 6.25 ; \mathrm{N}$, 14.47; Found: C, 68.76; H, 6.35; N, 14.83.

10-cyclohexyl-5-(3,4-dimethoxyphenyl)-2-(4-oxo-3-(pyridin-4-yl)3,4-dihydro-quinazolin-2-yl)-5,6,7,8,9,10-hexahydropyrimido[4,5-b] quinolin-4(3H)-one (9b)

Yellow crystals; Yield 58\%; m.p. 116-117 ${ }^{\circ} \mathrm{C}$; IR $\left(\mathrm{KBr}, \mathrm{cm}^{-1}\right): 3444(\mathrm{NH})$, 1722, $1718(\mathrm{C}=\mathrm{O}), 1650,1635,1629(\mathrm{C}=\mathrm{N}), 1217(\mathrm{OMe}) ;{ }^{1} \mathrm{H}-\mathrm{NMR}$ (500 MHz, DMSO-d $\left.\mathrm{d}_{6}, \delta / \mathrm{ppm}\right): 1.45\left(m, 2 \mathrm{H}, \mathrm{CH}_{2}\right), 1.50-1.81(m, 4 \mathrm{H}$, $\left.2 \mathrm{CH}_{2}\right), 1.52\left(t, 2 \mathrm{H}, \mathrm{CH}_{2}\right), 1.65\left(m, 2 \mathrm{H}, \mathrm{CH}_{2}\right), 1.74\left(m, 2 \mathrm{H}, \mathrm{CH}_{2}\right), 1.75-$ $2.20\left(q, 4 \mathrm{H}, 2 \mathrm{CH}_{2}\right), 1.82\left(t, 2 \mathrm{H}, \mathrm{CH}_{2}\right), 2.50(m, 1 \mathrm{H}, \mathrm{CH}), 3.79(s, 3 \mathrm{H}$, $\left.\mathrm{OCH}_{3}\right), 3.82\left(s, 3 \mathrm{H}, \mathrm{OCH}_{3}\right), 4.40(s, 1 \mathrm{H}, \mathrm{CH}), 6.61-8.70(m, 11 \mathrm{H}, \mathrm{Ar}-$ $\mathrm{H}), 11.30(\mathrm{~s}, 1 \mathrm{H}, \mathrm{NH})$; MS $(\mathrm{m} / z$, (relative abundance, $\%)): 642\left(\mathrm{M}^{+}\right.$, 35); Anal. Calcd. for $\mathrm{C}_{38} \mathrm{H}_{38} \mathrm{~N}_{6} \mathrm{O}_{4}: \mathrm{C}, 71.01 ; \mathrm{H}, 5.96 ; \mathrm{N}, 13.08$; Found: C, $71.45 ; \mathrm{H}, 5.78 ; \mathrm{N}, 13.30$.

2-(3-(4-bromophenyl)-4-oxo-3,4-dihydroquinazolin-2$y l)$-10-cyclohexyl-5-(3,4-dimethoxy-phenyl)-5, 6, 7, 8, 9, 10 hexahydropyrimido[4,5-b]quinolin-4(3H)-one (9c)

Yellow crystals; Yield 52\%; m.p. 136-137 ${ }^{\circ} \mathrm{C}$; IR $\left(\mathrm{KBr}, \mathrm{cm}^{-1}\right): 3450$ $(\mathrm{NH}), 1730,1717(\mathrm{C}=\mathrm{O}), 1624,1619(\mathrm{C}=\mathrm{N}), 1225(\mathrm{OMe}), 600(\mathrm{C}-\mathrm{Br})$; ${ }^{1} \mathrm{H}-\mathrm{NMR}\left(500 \mathrm{MHz}, \mathrm{DMSO}-\mathrm{d}_{6}, \delta / \mathrm{ppm}\right): 1.45\left(m, 2 \mathrm{H}, \mathrm{CH}_{2}\right), 1.50-1.81$ $\left(m, 4 \mathrm{H}, 2 \mathrm{CH}_{2}\right), 1.52\left(t, 2 \mathrm{H}, \mathrm{CH}_{2}\right), 1.65\left(m, 2 \mathrm{H}, \mathrm{CH}_{2}\right), 1.74(m, 2 \mathrm{H}$, $\left.\mathrm{CH}_{2}\right), 1.75-2.20\left(q, 4 \mathrm{H}, 2 \mathrm{CH}_{2}\right), 1.82\left(t, 2 \mathrm{H}, \mathrm{CH}_{2}\right), 2.50(m, 1 \mathrm{H}, \mathrm{CH}), 3.77$ $\left(s, 3 \mathrm{H}, \mathrm{OCH}_{3}\right), 3.80\left(s, 3 \mathrm{H}, \mathrm{OCH}_{3}\right), 4.40(s, 1 \mathrm{H}, \mathrm{CH}), 6.61-7.50(m, 11 \mathrm{H}$, $\operatorname{Ar}-\mathrm{H}), 11.12(s, 1 \mathrm{H}, \mathrm{NH}) ; \mathrm{MS}\left(\mathrm{m} / z\right.$, (relative abundance, \%)): $719\left(\mathrm{M}^{+}\right.$, 45), $722\left(\mathrm{M}^{+3}, 15\right)$; Anal. Calcd. for $\mathrm{C}_{39} \mathrm{H}_{38} \mathrm{BrN}_{5} \mathrm{O}_{4}: \mathrm{C}, 65.00 ; \mathrm{H}, 5.31 ; \mathrm{N}$, 9.72; Found: C, 65.34; H, 5.81; N, 9.48.

2-(3-(4-acetylphenyl)-4-oxo-3,4-dihydroquinazolin-2-yl)-10-cyclohexyl5-(3,4-di-methoxy-phenyl)-5, 6,7,8,9,10-hexahydropyrimido [4,5-b] quinolin-4(3H)-one (9d)

Yellow crystals; Yield $48 \%$; m.p. 126-127 ${ }^{\circ} \mathrm{C}$; IR $\left(\mathrm{KBr}, \mathrm{cm}^{-1}\right): 3445(\mathrm{NH})$, 1735, 1720, $1718(\mathrm{C}=\mathrm{O}), 1625,1619(\mathrm{C}=\mathrm{N}), 1220(\mathrm{OMe}) ;{ }^{1} \mathrm{H}-\mathrm{NMR}$ (500 MHz, DMSO-d $\left.\mathrm{d}_{6} \delta / \mathrm{ppm}\right): 1.45\left(m, 2 \mathrm{H}, \mathrm{CH}_{2}\right), 1.50-1.81(m, 4 \mathrm{H}$, $\left.2 \mathrm{CH}_{2}\right), 1.52\left(t, 2 \mathrm{H}, \mathrm{CH}_{2}\right), 1.65\left(m, 2 \mathrm{H}, \mathrm{CH}_{2}\right), 1.74\left(m, 2 \mathrm{H}, \mathrm{CH}_{2}\right), 1.75-$ $2.20\left(q, 4 \mathrm{H}, 2 \mathrm{CH}_{2}\right), 1.82\left(t, 2 \mathrm{H}, \mathrm{CH}_{2}\right), 2.50\left(s, 3 \mathrm{H}, \mathrm{CH}_{3}\right), 2.55(m, 1 \mathrm{H}$, $\mathrm{CH}), 3.78\left(s, 3 \mathrm{H}, \mathrm{OCH}_{3}\right), 3.80\left(s, 3 \mathrm{H}, \mathrm{OCH}_{3}\right), 4.40(s, 1 \mathrm{H}, \mathrm{CH}), 7.15-$ $8.80(\mathrm{~m}, 11 \mathrm{H}, \mathrm{Ar}-\mathrm{H}), 11.20(\mathrm{~s}, 1 \mathrm{H}, \mathrm{NH}) ; \mathrm{MS}(\mathrm{m} / z$, (relative abundance, \%)): $683\left(\mathrm{M}^{+}, 65\right)$; Anal. Calcd. for $\mathrm{C}_{41} \mathrm{H}_{41} \mathrm{~N}_{5} \mathrm{O}_{5}: \mathrm{C}, 72.02 ; \mathrm{H}, 6.04 ; \mathrm{N}$, 10.24; Found: C, 72.35; H, 6.48; N, 9.74.

Synthesis of 10-cyclohexyl-5-(3,4-dimethoxyphenyl)-2-(4-oxo3-(pyridin-2-yl)-3,4-dihydro-quinazolin-2-yl)-5, 6, 7, 8, 9, 10 hexahydropyrimido[4,5-b]quinolin-4(3H)-one (11)
A mixture of benzoxazinone 3 (3.93 g, 0.01 mole) and 2-aminopyridine or 3- aminopyridine ( $0.94 \mathrm{~g}, 0.01 \mathrm{~mole})$ was fused in an oil bath in presence of anhydrous $\mathrm{ZnCl}_{2}(1 \mathrm{~g})$ for $4 \mathrm{~h}$. The reaction mixture was triturated with ice $/ \mathrm{HCl}$. The formed solid product was filtered off, washed with water several times, dried and recrystallized from methanol to give 11 or 13 .

Yellow crystals; Yield 38\%; m.p. 136-137 ${ }^{\circ} \mathrm{C}$; IR $\left(\mathrm{KBr}, \mathrm{cm}^{-1}\right): 3477(\mathrm{NH})$, 1740, $1733(\mathrm{C}=\mathrm{O}), 1690,1683,1665(\mathrm{C}=\mathrm{N}), 1258(\mathrm{OMe}) ;{ }^{1} \mathrm{H}-\mathrm{NMR}$ (500 MHz, DMSO-d $\left.\mathrm{d}_{6}, \delta / \mathrm{ppm}\right): 1.45\left(m, 2 \mathrm{H}, \mathrm{CH}_{2}\right), 1.50-1.81(m, 4 \mathrm{H}$, $\left.2 \mathrm{CH}_{2}\right), 1.52\left(t, 2 \mathrm{H}, \mathrm{CH}_{2}\right), 1.65\left(m, 2 \mathrm{H}, \mathrm{CH}_{2}\right), 1.74\left(m, 2 \mathrm{H}, \mathrm{CH}_{2}\right), 1.75-$ $2.20\left(q, 4 \mathrm{H}, 2 \mathrm{CH}_{2}\right), 1.82\left(t, 2 \mathrm{H}, \mathrm{CH}_{2}\right), 2.50(\mathrm{~m}, 1 \mathrm{H}, \mathrm{CH}), 3.78(s, 3 \mathrm{H}$, $\left.\mathrm{OCH}_{3}\right), 3.81\left(s, 3 \mathrm{H}, \mathrm{OCH}_{3}\right), 4.40(s, 1 \mathrm{H}, \mathrm{CH}), 6.61-8.30(m, 11 \mathrm{H}, \mathrm{Ar}-$ $\mathrm{H}), 11.20(\mathrm{~s}, 1 \mathrm{H}, \mathrm{NH})$; MS $\left(\mathrm{m} / z\right.$, (relative abundance, \%)): $642\left(\mathrm{M}^{+}\right.$, 55); Anal. Calcd. for $\mathrm{C}_{38} \mathrm{H}_{38} \mathrm{~N}_{6} \mathrm{O}_{4}$ : C, 71.01; $\mathrm{H}, 5.96 ; \mathrm{N}, 13.08$; Found: $\mathrm{C}$, 71.23; H, 5.98; N, 13.41 .

Synthesis of 10-cyclohexyl-5-(3,4-dimethoxyphenyl)-2-(4-oxo3 -(pyridin-3-yl)-3,4-dihydro-quinazolin-2-yl) - 5, 6, 7, 8, 9, 10 hexahydropyrimido[4,5-b]quinolin-4 $(3 \mathrm{H})$-one (13)

Yellow crystals; Yield 38\%; m.p. 136-137 ${ }^{\circ} \mathrm{C}$; IR $\left(\mathrm{KBr}, \mathrm{cm}^{-1}\right): 3477(\mathrm{NH})$, 1740, $1733(\mathrm{C}=\mathrm{O}), 1690,1683,1665(\mathrm{C}=\mathrm{N}), 1258(\mathrm{OMe}) ;{ }^{1} \mathrm{H}-\mathrm{NMR}$ (500 MHz, DMSO-d $\left.\mathrm{d}_{6}, \delta / \mathrm{ppm}\right): 1.45\left(m, 2 \mathrm{H}, \mathrm{CH}_{2}\right), 1.50-1.81(m, 4 \mathrm{H}$, $\left.2 \mathrm{CH}_{2}\right), 1.52\left(t, 2 \mathrm{H}, \mathrm{CH}_{2}\right), 1.65\left(m, 2 \mathrm{H}, \mathrm{CH}_{2}\right), 1.74\left(m, 2 \mathrm{H}, \mathrm{CH}_{2}\right), 1.75-$ $2.20\left(q, 4 \mathrm{H}, 2 \mathrm{CH}_{2}\right), 1.82\left(t, 2 \mathrm{H}, \mathrm{CH}_{2}\right), 2.50(m, 1 \mathrm{H}, \mathrm{CH}), 3.77(s, 3 \mathrm{H}$, $\left.\mathrm{OCH}_{3}\right), 3.80\left(s, 3 \mathrm{H}, \mathrm{OCH}_{3}\right), 4.40(s, 1 \mathrm{H}, \mathrm{CH}), 6.61-8.30(m, 11 \mathrm{H}, \mathrm{Ar}-$ $\mathrm{H}), 11.20(\mathrm{~s}, 1 \mathrm{H}, \mathrm{NH})$; MS $\left(\mathrm{m} / z\right.$, (relative abundance, \%)): $642\left(\mathrm{M}^{+}\right.$, 55); Anal. Calcd. for $\mathrm{C}_{38} \mathrm{H}_{38} \mathrm{~N}_{6} \mathrm{O}_{4}$ : C, 71.01; H, 5.96; N, 13.08; Found: C, 71.23; H, 5.98; N, 13.41 .

Synthesis of 10-cyclohexyl-5-(3,4-dimethoxyphenyl)-2-(3-hydroxy-4-oxo3,4-dihydro-quinazolin-2-yl)-5,6,7,8,9,10-hexahydropyrimido[4,5-b] quinolin-4(3H)-one (15)

To a solution of benzoxazinone 3 (2.35 g, 0.006 mole) in EtOH (30 $\mathrm{mL}), \mathrm{NH}_{2} \mathrm{OH} . \mathrm{HCl}(0.417 \mathrm{~g}, 0.006$ mole $)$ and $\mathrm{CH}_{3} \mathrm{COONa}(0.49 \mathrm{~g}, 0.006$ mole) dissolved in the least amount of water. The reaction mixture was refluxed for $8 \mathrm{~h}$, cooled and then concentrated. The solid product was filtered off and recrystallized from ethanol to give $\mathbf{1 5 .}$

Yellowish brown crystals; Yield 65\%; m.p. $122-123{ }^{\circ} \mathrm{C}$; IR $\left(\mathrm{KBr}, \mathrm{cm}^{-1}\right)$ : $3633(\mathrm{OH}), 3392(\mathrm{NH}), 1738,1725(\mathrm{C}=\mathrm{O}), 1630,1612(\mathrm{C}=\mathrm{N}), 1251$ (OMe); ${ }^{1} \mathrm{H}-\mathrm{NMR}\left(500 \mathrm{MHz}, \mathrm{DMSO}-\mathrm{d}_{6}, \delta / \mathrm{ppm}\right): 1.45\left(m, 2 \mathrm{H}, \mathrm{CH}_{2}\right)$, 1.50-1.81 (m, 4H, $\left.2 \mathrm{CH}_{2}\right), 1.52\left(t, 2 \mathrm{H}, \mathrm{CH}_{2}\right), 1.65\left(m, 2 \mathrm{H}, \mathrm{CH}_{2}\right), 1.74(m$, $\left.2 \mathrm{H}, \mathrm{CH}_{2}\right), 1.75-2.20\left(q, 4 \mathrm{H}, 2 \mathrm{CH}_{2}\right), 1.82\left(t, 2 \mathrm{H}, \mathrm{CH}_{2}\right), 2.50(m, 1 \mathrm{H}, \mathrm{CH})$, $3.78\left(s, 3 \mathrm{H}, \mathrm{OCH}_{3}\right), 3.81\left(s, 3 \mathrm{H}, \mathrm{OCH}_{3}\right), 4.40(s, 1 \mathrm{H}, \mathrm{CH}), 6.10(s, 1 \mathrm{H}$, $\mathrm{OH}), 7.90-8.50(m, 7 \mathrm{H}, \mathrm{Ar}-\mathrm{H}), 10.89(\mathrm{~s}, 1 \mathrm{H}, \mathrm{NH}) ; \mathrm{MS}(\mathrm{m} / \mathrm{z}$, (relative abundance, \%)): $581\left(\mathrm{M}^{+}, 20\right)$; Anal. Calcd. for $\mathrm{C}_{33} \mathrm{H}_{35} \mathrm{~N}_{5} \mathrm{O}_{5}: \mathrm{C}, 68.14 ; \mathrm{H}$, 6.07; N, 12.04; Found: C, 68.20; H, 6.18; N, 12.26.

\section{Synthesis of compounds 17a-d}

To a solution of $9 \mathrm{a}(0.01$ mole $)$ in absolute $\mathrm{EtOH}(30 \mathrm{~mL})$ containing few drops of piperidine, appropriate aldehydes namely, p-methoxybenzaldehyde, p-fluorobenzaldehyde, p-nitrobenzaldehyde, or 2-thiophenealdehyde ( 0.01 mole) was added. The reaction mixture was refluxed for $5 \mathrm{~h}$, concentrated and left to cool. The precipitated product was filtered off and recrystallized from ethanol to give 17a-d.

10-cyclohexyl-5-(3,4-dimethoxyphenyl)-2-(3-((4-methoxybenzylidene) amino)-4-oxo-3,4-dihydro-quinazolin-2-yl)-5, 6, 7, 8, 9, 10 hexahydropyrimido[4,5-b]quinolin-4(3H)-one (17a)

Yellow crystals; Yield 65\%; m.p. 174- $175^{\circ} \mathrm{C}$; IR $\left(\mathrm{KBr}, \mathrm{cm}^{-1}\right): 3442(\mathrm{NH})$, 1733, $1722(\mathrm{C}=\mathrm{O}), 1650,1635,1619(\mathrm{C}=\mathrm{N}), 1226,1210,1205(\mathrm{OMe})$; ${ }^{1} \mathrm{H}-\mathrm{NMR}\left(500 \mathrm{MHz}, \mathrm{DMSO}-\mathrm{d}_{6}, \delta / \mathrm{ppm}\right): 1.45\left(m, 2 \mathrm{H}, \mathrm{CH}_{2}\right), 1.50-1.81$ $\left(m, 4 \mathrm{H}, 2 \mathrm{CH}_{2}\right), 1.52\left(t, 2 \mathrm{H}, \mathrm{CH}_{2}\right), 1.65\left(m, 2 \mathrm{H}, \mathrm{CH}_{2}\right), 1.74(m, 2 \mathrm{H}$, $\left.\mathrm{CH}_{2}\right), 1.75-2.20\left(q, 4 \mathrm{H}, 2 \mathrm{CH}_{2}\right), 1.82\left(t, 2 \mathrm{H}, \mathrm{CH}_{2}\right), 2.50(m, 1 \mathrm{H}, \mathrm{CH})$, 
$3.78\left(s, 3 \mathrm{H}, \mathrm{OCH}_{3}\right), 3.80\left(s, 3 \mathrm{H}, \mathrm{OCH}_{3}\right), 3.82\left(s, 3 \mathrm{H}, \mathrm{OCH}_{3}\right), 4.40(s$, $1 \mathrm{H}, \mathrm{CH}), 4.50(\mathrm{~s}, 1 \mathrm{H}, \mathrm{CH}=\mathrm{N}-), 6.81-7.50(\mathrm{~m}, 11 \mathrm{H}, \mathrm{Ar}-\mathrm{H}), 10.75(s, 1 \mathrm{H}$, $\mathrm{NH})$; MS $(\mathrm{m} / z$, (relative abundance, $\%)): 698\left(\mathrm{M}^{+}, 35\right)$; Anal. Calcd. for $\mathrm{C}_{41} \mathrm{H}_{42} \mathrm{~N}_{6} \mathrm{O}_{5}:$ C, 70.47; H, 6.06; N, 12.03; Found: C, 70.69; H, 6.56; N, 12.00 .

10-cyclohexyl-5-(3,4-dimethoxyphenyl)-2-(3-((4-fluorobenzylidene) amino)-4-oxo-3,4-dihydro-quinazolin-2-yl)-5,6,7,8,9,10hexahydropyrimido[4,5-b]quinolin-4(3H)-one (17b)

Yellow crystals; Yield 65\%; m.p. 169-170 ${ }^{\circ} \mathrm{C}$; IR $\left(\mathrm{KBr}, \mathrm{cm}^{-1}\right): 3441(\mathrm{NH})$, 1733, $1723(\mathrm{C}=\mathrm{O}), 1632,1623,1615(\mathrm{C}=\mathrm{N}), 1220(\mathrm{OMe}) ;{ }^{1} \mathrm{H}-\mathrm{NMR}$ (500 MHz, DMSO-d,$\delta /$ ppm): $1.45\left(m, 2 \mathrm{H}, \mathrm{CH}_{2}\right), 1.50-1.81(m, 4 \mathrm{H}, 2$ $\left.\mathrm{CH}_{2}\right), 1.52\left(t, 2 \mathrm{H}, \mathrm{CH}_{2}\right), 1.65\left(m, 2 \mathrm{H}, \mathrm{CH}_{2}\right), 1.74\left(m, 2 \mathrm{H}, \mathrm{CH}_{2}\right), 1.75-2.20$ $\left(q, 4 \mathrm{H}, 2 \mathrm{CH}_{2}\right), 1.82\left(t, 2 \mathrm{H}, \mathrm{CH}_{2}\right), 2.50(m, 1 \mathrm{H}, \mathrm{CH}), 3.77\left(s, 3 \mathrm{H}, \mathrm{OCH}_{3}\right)$, $3.80\left(s, 3 \mathrm{H}, \mathrm{OCH}_{3}\right), 4.11(s, 1 \mathrm{H}, \mathrm{CH}=\mathrm{N}-), 4.40(s, 1 \mathrm{H}, \mathrm{CH}), 7.20-7.75$ $(m, 11 \mathrm{H}, \operatorname{Ar}-\mathrm{H}), 11.20(s, 1 \mathrm{H}, \mathrm{NH})$; $\mathrm{MS}(\mathrm{m} / z$, (relative abundance, $\%)$ ): $686\left(\mathrm{M}^{+}, 45\right)$; Anal. Calcd. for $\mathrm{C}_{40} \mathrm{H}_{39} \mathrm{FN}_{6} \mathrm{O}_{4}: \mathrm{C}, 69.95 ; \mathrm{H}, 5.72 ; \mathrm{N}, 12.24$; Found: C, 69.97; H, 5.36; N, 12.18 .

10-cyclohexyl-5-(3,4-dimethoxyphenyl)-2-(3-((4-nitrobenzylidene) amino)-4-oxo-3,4-dihydro-quinazolin-2-yl)-5, 6, 7, 8, 9, 10 hexahydropyrimido[4,5-b]quinolin-4(3H)-one (17c)

Yellow crystals; Yield 53\%; m.p. 106-107 ${ }^{\circ} \mathrm{C}$; IR $\left(\mathrm{KBr}, \mathrm{cm}^{-1}\right): 3448(\mathrm{NH})$, 1753, $1740(\mathrm{C}=\mathrm{O}), 1642,1622,1610(\mathrm{C}=\mathrm{N}), 1226(\mathrm{OMe}) ;{ }^{1} \mathrm{H}-\mathrm{NMR}$ (500 MHz, DMSO-d,$\delta / \mathrm{ppm}): 1.45\left(m, 2 \mathrm{H}, \mathrm{CH}_{2}\right), 1.50-1.81(m, 4 \mathrm{H}, 2$ $\left.\mathrm{CH}_{2}\right), 1.52\left(t, 2 \mathrm{H}, \mathrm{CH}_{2}\right), 1.65\left(m, 2 \mathrm{H}, \mathrm{CH}_{2}\right), 1.74\left(m, 2 \mathrm{H}, \mathrm{CH}_{2}\right), 1.75-2.20$ $\left(q, 4 \mathrm{H}, 2 \mathrm{CH}_{2}\right), 1.82\left(t, 2 \mathrm{H}, \mathrm{CH}_{2}\right), 2.50(m, 1 \mathrm{H}, \mathrm{CH}), 3.78\left(s, 3 \mathrm{H}, \mathrm{OCH}_{3}\right)$, $3.81\left(s, 3 \mathrm{H}, \mathrm{OCH}_{3}\right), 4.20(s, 1 \mathrm{H}, \mathrm{CH}=\mathrm{N}-), 4.40(s, 1 \mathrm{H}, \mathrm{CH}), 7.20-7.75$ $(m, 11 \mathrm{H}, \operatorname{Ar}-\mathrm{H}), 11.20(s, 1 \mathrm{H}, \mathrm{NH}) ; \mathrm{MS}(\mathrm{m} / z$, (relative abundance, $\%))$ : $713\left(\mathrm{M}^{+}, 40\right)$; Anal. Calcd. for $\mathrm{C}_{40} \mathrm{H}_{39} \mathrm{~N}_{7} \mathrm{O}_{6}: \mathrm{C}, 67.31 ; \mathrm{H}, 5.51 ; \mathrm{N}, 13.74$; Found: C, 67.81; H, 5.39; N, 13.41 .

10-cyclohexyl-5-(3,4-dimethoxyphenyl)-2-(4-oxo-3-((thiophen2-ylmethylene)amino)-3,4-dihydroquinazolin-2-yl)-5, 6, 7, 8,9,10hexahydropyrimido[4,5-b]quinolin-4(3H)-one (17d)

Yellow crystals; Yield 50\%; m.p. 116-118 ${ }^{\circ} \mathrm{C}$; IR $\left(\mathrm{KBr}, \mathrm{cm}^{-1}\right): 3450(\mathrm{NH})$, 1720, $1712(\mathrm{C}=\mathrm{O}), 1640,1638,1622(\mathrm{C}=\mathrm{N}), 1225(\mathrm{OMe}) ;{ }^{1} \mathrm{H}-\mathrm{NMR}$ (500 MHz, DMSO-d $\left.\mathrm{d}_{6} \delta / \mathrm{ppm}\right): 1.45\left(m, 2 \mathrm{H}, \mathrm{CH}_{2}\right), 1.50-1.81(m, 4 \mathrm{H}$, $\left.2 \mathrm{CH}_{2}\right), 1.52\left(t, 2 \mathrm{H}, \mathrm{CH}_{2}\right), 1.65\left(m, 2 \mathrm{H}, \mathrm{CH}_{2}\right), 1.74\left(m, 2 \mathrm{H}, \mathrm{CH}_{2}\right), 1.75-$ $2.20\left(q, 4 \mathrm{H}, 2 \mathrm{CH}_{2}\right), 1.82\left(t, 2 \mathrm{H}, \mathrm{CH}_{2}\right), 2.50(m, 1 \mathrm{H}, \mathrm{CH}), 3.78(s, 3 \mathrm{H}$, $\left.\mathrm{OCH}_{3}\right), 3.80\left(s, 3 \mathrm{H}, \mathrm{OCH}_{3}\right), 4.40(s, 1 \mathrm{H}, \mathrm{CH}), 4.60(s, 1 \mathrm{H}, \mathrm{CH}=\mathrm{N}-)$, 7.50-7.95 ( $m, 10 \mathrm{H}, \mathrm{Ar}-\mathrm{H}$, thiophene $\mathrm{H}), 11.20(\mathrm{~s}, 1 \mathrm{H}, \mathrm{NH})$; $\mathrm{MS}(\mathrm{m} / z$, (relative abundance, \%)): $674\left(\mathrm{M}^{+}, 40\right)$; Anal. Calcd. for $\mathrm{C}_{38} \mathrm{H}_{38} \mathrm{~N}_{6} \mathrm{O}_{4} \mathrm{~S}: \mathrm{C}$, 67.63; H, 5.68; N, 12.45; Found: C, 67.53; H, 5.21; N, 12.18 .

\section{Synthesis of $19,21 a, b$}

A solution of quinazolinone 9a (4.07 g, 0.01 mole), acetyl chloride, benzoyl chloride, and $p$-chlorobenzoyl chloride ( 0.02 mole) in dry pyridine $(30 \mathrm{~mL})$ was heated under reflux for 3 hours. The reaction mixture was cooled, then poured over ice/ $\mathrm{HCl}$ and the solid that separated out was filtered off, washed with water several times, dried and then recrystallized from methanol to afford 19 and $21 \mathrm{a}, \mathrm{b}$, respectively.

$N$-acetyl-N-(2-(10-cyclohexyl-5-(3,4-dimethoxyphenyl)-4-oxo$3,4,5,6,7,8,9,10$-octa-hydro-pyrimido [4,5-b]quinolin-2-yl)-4oxoquinazolin-3(4H)-yl)acetamide (19)

Yellow crystals; Yield 63\%; m.p. 133-134 ${ }^{\circ} \mathrm{C}$; IR $\left(\mathrm{KBr}, \mathrm{cm}^{-1}\right): 3390$ $(\mathrm{NH}), 1758,1743,1735,1720(\mathrm{C}=\mathrm{O}), 1632,1618(\mathrm{C}=\mathrm{N}), 1217(\mathrm{OMe})$; ${ }^{1} \mathrm{H}-\mathrm{NMR}\left(500 \mathrm{MHz}, \mathrm{DMSO}-\mathrm{d}_{6}, \delta / \mathrm{ppm}\right): 1.45\left(\mathrm{~m}, 2 \mathrm{H}, \mathrm{CH}_{2}\right), 1.50-$ $1.81\left(m, 4 \mathrm{H}, 2 \mathrm{CH}_{2}\right), 1.52\left(t, 2 \mathrm{H}, \mathrm{CH}_{2}\right), 1.65\left(m, 2 \mathrm{H}, \mathrm{CH}_{2}\right), 1.74(m$, $\left.2 \mathrm{H}, \mathrm{CH}_{2}\right), 1.75-2.20\left(q, 4 \mathrm{H}, 2 \mathrm{CH}_{2}\right), 1.82\left(t, 2 \mathrm{H}, \mathrm{CH}_{2}\right), 2.40(s, 6 \mathrm{H}, 2$ $\left.\mathrm{COCH}_{3}\right), 2.50(m, 1 \mathrm{H}, \mathrm{CH}), 3.78\left(s, 3 \mathrm{H}, \mathrm{OCH}_{3}\right), 3.80\left(s, 3 \mathrm{H}, \mathrm{OCH}_{3}\right)$, $4.40(s, 1 \mathrm{H}, \mathrm{CH}), 6.61-7.90(m, 7 \mathrm{H}, \mathrm{Ar}-\mathrm{H}), 11.20(s, 1 \mathrm{H}, \mathrm{NH})$; $\mathrm{MS}(\mathrm{m} / z$, (relative abundance, \%)): $664\left(\mathrm{M}^{+}, 30\right)$; Anal. Calcd. for $\mathrm{C}_{37} \mathrm{H}_{40} \mathrm{~N}_{6} \mathrm{O}_{6}: \mathrm{C}$, $66.85 ; \mathrm{H}, 6.07$; N, 12.64; Found: C, 66.55; H, 6.10; N, 12.45.

$\mathrm{N}$-benzoyl-N-(2-(10-cyclohexyl-5-(3,4-dimethoxyphenyl)-4-oxo3, 4,5, 6, 7, 8,9, 10-octa-hydro-pyrimido[4,5-b]quinolin-2-yl)-4oxoquinazolin-3(4H)-yl)benzamide (21a)

Yellow crystals; Yield 50\%; m.p. 112-113 ${ }^{\circ} \mathrm{C}$; IR $\left(\mathrm{KBr}, \mathrm{cm}^{-1}\right): 3453$ $(\mathrm{NH}), 1750,1731,1728,1718(\mathrm{C}=\mathrm{O}), 1633,1619(\mathrm{C}=\mathrm{N}), 1218(\mathrm{OMe})$; ${ }^{1} \mathrm{H}-\mathrm{NMR}\left(500 \mathrm{MHz}, \mathrm{DMSO}-\mathrm{d}_{6}, \delta / \mathrm{ppm}\right): 1.45\left(\mathrm{~m}, 2 \mathrm{H}, \mathrm{CH}_{2}\right), 1.50-1.81$ $\left(m, 4 \mathrm{H}, 2 \mathrm{CH}_{2}\right), 1.52\left(t, 2 \mathrm{H}, \mathrm{CH}_{2}\right), 1.65\left(m, 2 \mathrm{H}, \mathrm{CH}_{2}\right), 1.74(m, 2 \mathrm{H}$, $\left.\mathrm{CH}_{2}\right), 1.75-2.20\left(q, 4 \mathrm{H}, 2 \mathrm{CH}_{2}\right), 1.82\left(t, 2 \mathrm{H}, \mathrm{CH}_{2}\right), 2.50(m, 1 \mathrm{H}, \mathrm{CH}), 3.77$ $\left(s, 3 \mathrm{H}, \mathrm{OCH}_{3}\right), 3.80\left(s, 3 \mathrm{H}, \mathrm{OCH}_{3}\right), 4.40(s, 1 \mathrm{H}, \mathrm{CH}), 6.61-8.20(m, 17 \mathrm{H}$, Ar-H), $11.20(s, 1 \mathrm{H}, \mathrm{NH})$; MS $(\mathrm{m} / z$, (relative abundance, \% $)): 788\left(\mathrm{M}^{+}\right.$, 20); Anal. Calcd. for $\mathrm{C}_{47} \mathrm{H}_{44} \mathrm{~N}_{6} \mathrm{O}_{6}: \mathrm{C}, 71.56 ; \mathrm{H}, 5.62 ; \mathrm{N}, 10.65$; Found: $\mathrm{C}$, $71.83 ; \mathrm{H}, 5.30 ; \mathrm{N}, 10.54$.

4-chloro-N-(4-chlorobenzoyl)-N-(2-(10-cyclohexyl-5-(3,4dimethoxyphenyl)-4-oxo-3,4,5,6,7,8,9, 10-octahydropyrimido[4,5-b] quinolin-2-yl)-4-oxoquinazolin-3(4H)-yl)benzamide (21b)

Yellow crystals; Yield 50\%; m.p. 129-130 ${ }^{\circ} \mathrm{C}$; IR $\left(\mathrm{KBr}, \mathrm{cm}^{-1}\right): 3501(\mathrm{NH})$, 1744, 1727, 1720, $1718(\mathrm{C}=\mathrm{O}), 1650,1635(\mathrm{C}=\mathrm{N}), 1225(\mathrm{OMe}), 750,733$ (C-Cl); ${ }^{1} \mathrm{H}-\mathrm{NMR}\left(500 \mathrm{MHz}, \mathrm{DMSO}-\mathrm{d}_{6}, \delta / \mathrm{ppm}\right): 1.45\left(m, 2 \mathrm{H}, \mathrm{CH}_{2}\right)$, 1.50-1.81 ( $\left.m, 4 \mathrm{H}, 2 \mathrm{CH}_{2}\right), 1.52\left(t, 2 \mathrm{H}, \mathrm{CH}_{2}\right), 1.65\left(m, 2 \mathrm{H}, \mathrm{CH}_{2}\right), 1.74(m$, $\left.2 \mathrm{H}, \mathrm{CH}_{2}\right), 1.75-2.20\left(q, 4 \mathrm{H}, 2 \mathrm{CH}_{2}\right), 1.82\left(t, 2 \mathrm{H}, \mathrm{CH}_{2}\right), 2.50(m, 1 \mathrm{H}, \mathrm{CH})$, $3.76\left(s, 3 \mathrm{H}, \mathrm{OCH}_{3}\right), 3.80\left(s, 3 \mathrm{H}, \mathrm{OCH}_{3}\right), 4.40(s, 1 \mathrm{H}, \mathrm{CH}), 6.61-8.20(m$, $15 \mathrm{H}, \mathrm{Ar}-\mathrm{H}), 11.10(\mathrm{~s}, 1 \mathrm{H}, \mathrm{NH}) ; \mathrm{MS}(\mathrm{m} / z$, (relative abundance, $\%)): 857$ $\left(\mathrm{M}^{+}, 20\right)$; Anal. Calcd. for $\mathrm{C}_{47} \mathrm{H}_{42} \mathrm{Cl}_{2} \mathrm{~N}_{6} \mathrm{O}_{6}: \mathrm{C}, 65.81 ; \mathrm{H}, 4.94 ; \mathrm{N}, 9.80$; Found: C, 65.51; H, 4.92; N, 9.83.

\section{Synthesis of $23 a, b$}

A mixture of quinazolinone $7 \mathrm{a}(0.01$ mole) and maleic anhydride or phthalic anhydride $(0.01$ mole) was fused in an oil bath at for 6 hrs. The reaction mixture was triturated with ice $/ \mathrm{HCl}$. The solid product was filtered off, washed with water several times, dried and then recrystallized from ethanol affording 23a,b.

1-(2-(10-cyclohexyl-5-(3,4-dimethoxyphenyl)-4-oxo-3, 4,5, 6, 7,8,9,10octahydro-pyrimido[4,5-b]quinolin-2-yl)-4-oxoquinazolin-3(4H)-yl)1 H-pyrrole-2,5-dione (23a)

Yellow crystals; Yield 52\%; m.p. 143-145 ${ }^{\circ} \mathrm{C}$; IR $\left(\mathrm{KBr}, \mathrm{cm}^{-1}\right)$ : 3444 $(\mathrm{NH}), 1745,1730,1722,1715(\mathrm{C}=\mathrm{O}), 1630,1617(\mathrm{C}=\mathrm{N}), 1217(\mathrm{OMe})$; ${ }^{1} \mathrm{H}-\mathrm{NMR}\left(500 \mathrm{MHz}, \mathrm{DMSO}-\mathrm{d}_{6}, \delta / \mathrm{ppm}\right): 1.45\left(m, 2 \mathrm{H}, \mathrm{CH}_{2}\right), 1.50-1.81$ $\left(m, 4 \mathrm{H}, 2 \mathrm{CH}_{2}\right), 1.52\left(t, 2 \mathrm{H}, \mathrm{CH}_{2}\right), 1.65\left(m, 2 \mathrm{H}, \mathrm{CH}_{2}\right), 1.74(m, 2 \mathrm{H}$, $\left.\mathrm{CH}_{2}\right), 1.75-2.20\left(q, 4 \mathrm{H}, 2 \mathrm{CH}_{2}\right), 1.82\left(t, 2 \mathrm{H}, \mathrm{CH}_{2}\right), 2.50(m, 1 \mathrm{H}, \mathrm{CH})$, $3.77\left(s, 3 \mathrm{H}, \mathrm{OCH}_{3}\right), 3.80\left(s, 3 \mathrm{H}, \mathrm{OCH}_{3}\right), 4.40(s, 1 \mathrm{H}, \mathrm{CH}), 5.30(d d$, $2 \mathrm{H}, \mathrm{CH}=\mathrm{CH}), 6.61-7.90(m, 7 \mathrm{H}, \mathrm{Ar}-\mathrm{H}), 11.20(\mathrm{~s}, 1 \mathrm{H}, \mathrm{NH}) ; \mathrm{MS}(\mathrm{m} / z$, (relative abundance, \%)): $660\left(\mathrm{M}^{+}, 60\right)$; Anal. Calcd. for $\mathrm{C}_{37} \mathrm{H}_{36} \mathrm{~N}_{6} \mathrm{O}_{6}: \mathrm{C}$, 67.26; H, 5.49; N, 12.72; Found: C, 67.15; H, 5.30; N, 12.91.

2-(2-(10-cyclohexyl-5-(3,4-dimethoxyphenyl)-4-oxo-3,4,5,6,7,8,9,10octahydro-pyrimido[4,5-b]quinolin-2-yl)-4-oxoquinazolin-3(4H)-yl) isoindoline-1,3-dione (23b)

Yellow crystals; Yield 65\%; m.p. 162-163 ${ }^{\circ} \mathrm{C}$; IR $\left(\mathrm{KBr}, \mathrm{cm}^{-1}\right): 3443$ $(\mathrm{NH}), 1739,1725,1720,1710(\mathrm{C}=\mathrm{O}), 1650,1644(\mathrm{C}=\mathrm{N}), 1217(\mathrm{OMe})$; ${ }^{1} \mathrm{H}-\mathrm{NMR}\left(500 \mathrm{MHz}, \mathrm{DMSO}-\mathrm{d}_{6}, \delta / \mathrm{ppm}\right): 1.45\left(m, 2 \mathrm{H}, \mathrm{CH}_{2}\right), 1.50-1.81$ $\left(m, 4 \mathrm{H}, 2 \mathrm{CH}_{2}\right), 1.52\left(t, 2 \mathrm{H}, \mathrm{CH}_{2}\right), 1.65\left(m, 2 \mathrm{H}, \mathrm{CH}_{2}\right), 1.74(m, 2 \mathrm{H}$, $\left.\mathrm{CH}_{2}\right), 1.75-2.20\left(q, 4 \mathrm{H}, 2 \mathrm{CH}_{2}\right), 1.82\left(t, 2 \mathrm{H}, \mathrm{CH}_{2}\right), 2.50(m, 1 \mathrm{H}, \mathrm{CH}), 3.79$ $\left(s, 3 \mathrm{H}, \mathrm{OCH}_{3}\right), 3.81\left(s, 3 \mathrm{H}, \mathrm{OCH}_{3}\right), 4.40(s, 1 \mathrm{H}, \mathrm{CH}), 6.61-8.80(m, 11 \mathrm{H}$, Ar-H), $11.05(s, 1 \mathrm{H}, \mathrm{NH})$; MS $\left(\mathrm{m} / z\right.$, (relative abundance, \%)): $710\left(\mathrm{M}^{+}\right.$, 55); Anal. Calcd. for $\mathrm{C}_{41} \mathrm{H}_{38} \mathrm{~N}_{6} \mathrm{O}_{6}: \mathrm{C}, 69.28 ; \mathrm{H}, 5.39 ; \mathrm{N}, 11.82$; Found: $\mathrm{C}$, $69.46 ; \mathrm{H}, 5.71 ; \mathrm{N}, 11.38$. 


\section{Synthesis of compound 25a,b}

A mixture of 17a,b (0.01 mole), chloroacetyl chloride (1.13 g, 0.01 mole) and triethyl amine (5 drops) in dry dioxane $(30 \mathrm{~mL})$ was heated under reflux for 8 hours. The solid product that separated on cooling was filtered off, dried and recrystallized from ethanol to give 25a,b.

2-(3-(3-chloro-2-(4-methoxyphenyl)-4-oxoazetidin-1-yl)-4-oxo-3,4dihydro-quinazolin-2-yl)-10-cyclohexyl-5-(3,4-dimethoxyphenyl)5,6,7,8,9,10-hexahydropyrimido[4,5-b]quinolin-4(3H)-one (25a)

Yellow crystals; Yield $60 \%$; m.p. 156-158 ${ }^{\circ} \mathrm{C}$; IR $\left(\mathrm{KBr}, \mathrm{cm}^{-1}\right): 3444(\mathrm{NH})$, 1750, 1738, $1720(\mathrm{C}=\mathrm{O}), 1644,1620(\mathrm{C}=\mathrm{N}), 1219(\mathrm{OMe}), 750(\mathrm{C}-\mathrm{Cl})$; ${ }^{1} \mathrm{H}-\mathrm{NMR}\left(500 \mathrm{MHz}, \mathrm{DMSO}-\mathrm{d}_{6}, \delta / \mathrm{ppm}\right): 1.45\left(m, 2 \mathrm{H}, \mathrm{CH}_{2}\right), 1.50-1.81$ $\left(m, 4 \mathrm{H}, 2 \mathrm{CH}_{2}\right), 1.52\left(t, 2 \mathrm{H}, \mathrm{CH}_{2}\right), 1.65\left(m, 2 \mathrm{H}, \mathrm{CH}_{2}\right), 1.74(m, 2 \mathrm{H}$, $\left.\mathrm{CH}_{2}\right), 1.75-2.20\left(q, 4 \mathrm{H}, 2 \mathrm{CH}_{2}\right), 1.82\left(t, 2 \mathrm{H}, \mathrm{CH}_{2}\right), 2.50(m, 1 \mathrm{H}, \mathrm{CH})$, $2.72\left(s, 3 \mathrm{H}, \mathrm{OCH}_{3}\right), 2.90(d, 1 \mathrm{H}, \mathrm{CH}), 3.50(d, 1 \mathrm{H}, \mathrm{CH}-\mathrm{Cl}), 4.10(s, 6 \mathrm{H}$, $\left.2 \mathrm{OCH}_{3}\right), 4.40(s, 1 \mathrm{H}, \mathrm{CH}), 6.20(s, 1 \mathrm{H}, \mathrm{NH}), 6.98-7.90(m, 11 \mathrm{H}, \mathrm{Ar}-\mathrm{H})$; MS ( $m / z$, (relative abundance, \%)): $775\left(\mathrm{M}^{+}, 45\right), 777\left(\mathrm{M}^{+2}, 15\right)$; Anal. Calcd. for $\mathrm{C}_{43} \mathrm{H}_{43} \mathrm{ClN}_{6} \mathrm{O}_{6}$ : C, 66.61; H, 5.59; N, 10.84; Found: C, 66.34; $\mathrm{H}, 5.37 ; \mathrm{N}, 10.85$.

2-(3-(3-chloro-2-(4-fluorophenyl)-4-oxoazetidin-1-yl)-4-oxo-3,4dihydroquinazolin-2-yl)-10-cyclohexyl-5-(3,4-dimethoxyphenyl)5,6,7,8,9,10-hexahydropyrimido[4,5-b]quinolin-4(3H)-one (25b)

Yellow crystals; Yield 50\%; m.p. 102-103이 $\mathrm{C}$; $\left(\mathrm{KBr}, \mathrm{cm}^{-1}\right): 3444(\mathrm{NH})$, 1750, 1738, $1720(\mathrm{C}=\mathrm{O}), 1644,1620(\mathrm{C}=\mathrm{N}), 1219(\mathrm{OMe}), 750(\mathrm{C}-\mathrm{Cl})$; ${ }^{1} \mathrm{H}-\mathrm{NMR}\left(500 \mathrm{MHz}, \mathrm{DMSO}-\mathrm{d}_{6}, \delta / \mathrm{ppm}\right): 1.45\left(m, 2 \mathrm{H}, \mathrm{CH}_{2}\right), 1.50-1.81$ $\left(m, 4 \mathrm{H}, 2 \mathrm{CH}_{2}\right), 1.52\left(t, 2 \mathrm{H}, \mathrm{CH}_{2}\right), 1.65\left(m, 2 \mathrm{H}, \mathrm{CH}_{2}\right), 1.74(m, 2 \mathrm{H}$, $\left.\mathrm{CH}_{2}\right), 1.75-2.20\left(q, 4 \mathrm{H}, 2 \mathrm{CH}_{2}\right), 1.82\left(t, 2 \mathrm{H}, \mathrm{CH}_{2}\right), 2.50(m, 1 \mathrm{H}, \mathrm{CH})$, $2.90(d, 1 \mathrm{H}, \mathrm{CH}), 3.50(d, 1 \mathrm{H}, \mathrm{CH}-\mathrm{Cl}), 3.78\left(s, 3 \mathrm{H}, \mathrm{OCH}_{3}\right), 3.80(s, 3 \mathrm{H}$, $\left.\mathrm{OCH}_{3}\right), 3.82\left(s, 3 \mathrm{H}, \mathrm{OCH}_{3}\right), 4.40(s, 1 \mathrm{H}, \mathrm{CH}),, 6.98-7.90(m, 11 \mathrm{H}, \mathrm{Ar}-$ $\mathrm{H}), 10.95(\mathrm{~s}, 1 \mathrm{H}, \mathrm{NH}) ; \mathrm{MS}\left(\mathrm{m} / z\right.$, (relative abundance, \%)): $763\left(\mathrm{M}^{+}, 40\right)$; Anal. Calcd. for $\mathrm{C}_{42} \mathrm{H}_{40} \mathrm{ClFN}_{6} \mathrm{O}_{5}: \mathrm{C}, 66.09 ; \mathrm{H}, 5.28 ; \mathrm{N}, 11.01$; Found: C, 65.66; H, 5.07; N, 11.51 .

\section{Synthesis of $27 a, b$ and $29 a, b$}

A mixture of compound 17a,b (4.95 g, 0.01 mole) and thioglycolic acid (26) or thiosalicylic acid (28) (0.01 mole) in dry benzene $(20 \mathrm{~mL})$ was added drop wise with stirring at room temperature for 1 hour. The reaction mixture was heated under reflux for 6 hours, cooled and the precipitated product was filtered off and recrystallized from ethanol to give the desired products $27 \mathrm{a}, \mathrm{b}$ and $29 \mathrm{a}, \mathrm{b}$ respectively.

3-(2-(10-cyclohexyl-5-(3,4-dimethoxyphenyl)-4-oxo-3,4,5,6,7,8,9,10octahydro-pyrimido[4,5-b]quinolin-2-yl)-4-oxoquinazolin-3(4H)-yl)-2(4-methoxyphenyl)thiazolidin-4-one (27a)

Yellow crystals; Yield 58\%; m.p. 126-128 ${ }^{\circ} \mathrm{C}$; IR $\left(\mathrm{KBr}, \mathrm{cm}^{-1}\right): 3414(\mathrm{NH})$, 1780, 1737, $1720(\mathrm{C}=\mathrm{O}), 1630,1619(\mathrm{C}=\mathrm{N}), 1223(\mathrm{OMe}) ;{ }^{1} \mathrm{H}-\mathrm{NMR}$ (500 MHz, DMSO-d $\left.\mathrm{d}_{6}, \delta / \mathrm{ppm}\right): 1.45\left(m, 2 \mathrm{H}, \mathrm{CH}_{2}\right), 1.50-1.81(m, 4 \mathrm{H}$, $\left.2 \mathrm{CH}_{2}\right), 1.52\left(t, 2 \mathrm{H}, \mathrm{CH}_{2}\right), 1.65\left(m, 2 \mathrm{H}, \mathrm{CH}_{2}\right), 1.74\left(m, 2 \mathrm{H}, \mathrm{CH}_{2}\right), 1.75-$ $2.20\left(q, 4 \mathrm{H}, 2 \mathrm{CH}_{2}\right), 1.82\left(t, 2 \mathrm{H}, \mathrm{CH}_{2}\right), 2.50(m, 1 \mathrm{H}, \mathrm{CH}), 3.20(s, 1 \mathrm{H}$, $\mathrm{CH}$, thiazole $), 3.76\left(s, 3 \mathrm{H}, \mathrm{OCH}_{3}\right), 3.79\left(s, 3 \mathrm{H}, \mathrm{OCH}_{3}\right), 3.81(s, 3 \mathrm{H}$, $\left.\mathrm{OCH}_{3}\right), 4.40(s, 1 \mathrm{H}, \mathrm{CH}), 4.45\left(s, 2 \mathrm{H}, \mathrm{CH}_{2}\right.$, thiazole $), 6.61-7.10(m, 11 \mathrm{H}$, $\operatorname{Ar}-\mathrm{H}), 10.95(s, 1 \mathrm{H}, \mathrm{NH}) ; \mathrm{MS}\left(\mathrm{m} / \mathrm{z}\right.$, (relative abundance, \%)): $772\left(\mathrm{M}^{+}\right.$, 35); Anal. Calcd. for $\mathrm{C}_{43} \mathrm{H}_{44} \mathrm{~N}_{6} \mathrm{O}_{6} \mathrm{~S}$ : C, 66.82; H, 5.74; N, 10.87; Found: C, 66.63; H, 5.46; N, 10.86 .

3-(2-(10-cyclohexyl-5-(3,4-dimethoxyphenyl)-4-oxo-3,4,5,6,7,8,9,10octahydro-pyrimido[4,5-b]quinolin-2-yl)-4-oxoquinazolin-3(4H)-yl)-2(4-fluorophenyl)thiazolidin-4-one (27b)

Yellow crystals; Yield 55\%; m.p. $135-137^{\circ} \mathrm{C}$; IR $\left(\mathrm{KBr}, \mathrm{cm}^{-1}\right): 3414(\mathrm{NH})$, 1780, 1737, $1720(\mathrm{C}=\mathrm{O}), 1630,1619(\mathrm{C}=\mathrm{N}), 1223(\mathrm{OMe}) ;{ }^{1} \mathrm{H}-\mathrm{NMR}$ (500 MHz, DMSO-d $\left.\mathrm{d}_{6}, \delta / \mathrm{ppm}\right): 1.45\left(m, 2 \mathrm{H}, \mathrm{CH}_{2}\right), 1.50-1.81(m, 4 \mathrm{H}$, $\left.2 \mathrm{CH}_{2}\right), 1.52\left(t, 2 \mathrm{H}, \mathrm{CH}_{2}\right), 1.65\left(m, 2 \mathrm{H}, \mathrm{CH}_{2}\right), 1.74\left(m, 2 \mathrm{H}, \mathrm{CH}_{2}\right), 1.75-$ $2.20\left(q, 4 \mathrm{H}, 2 \mathrm{CH}_{2}\right), 1.82\left(t, 2 \mathrm{H}, \mathrm{CH}_{2}\right), 2.50(m, 1 \mathrm{H}, \mathrm{CH}), 3.20(s, 1 \mathrm{H}$, $\mathrm{CH}$, thiazole $), 3.77\left(s, 3 \mathrm{H}, \mathrm{OCH}_{3}\right), 3.80\left(s, 3 \mathrm{H}, \mathrm{OCH}_{3}\right), 4.40(s, 1 \mathrm{H}$, $\mathrm{CH}), 4.45\left(s, 2 \mathrm{H}, \mathrm{CH}_{2}\right.$, thiazole $), 6.61-7.10(m, 11 \mathrm{H}, \mathrm{Ar}-\mathrm{H}), 11.05(s$, $1 \mathrm{H}, \mathrm{NH})$; MS $\left(\mathrm{m} / z\right.$, (relative abundance, \%)): $760\left(\mathrm{M}^{+}, 35\right)$; Anal. Calcd. for $\mathrm{C}_{42} \mathrm{H}_{41 \mathrm{~F}} \mathrm{~N}_{6} \mathrm{O}_{5} \mathrm{~S}$ : C, 66.30; H, 5.43; N, 11.05; Found: C, 66.16; H, 5.46; $\mathrm{N}, 11.28$.

3-(2-(10-cyclohexyl-5-(3,4-dimethoxyphenyl)-4-oxo-3,4,5,6,7,8,9,10octahydro-pyrimido[4,5-b]quinolin-2-yl)-4-oxoquinazolin-3(4H)-yl)-2(4-methoxyphenyl)-2H-benzo[e][1,3]thiazin-4(3H)-one (29a)

Yellowish brown crystals; Yield 68\%; m.p. $150-151{ }^{\circ} \mathrm{C}$; IR $\left(\mathrm{KBr}, \mathrm{cm}^{-1}\right)$ : $3396(\mathrm{NH}), 1740,1735,1720(\mathrm{C}=\mathrm{O}), 1690,1650(\mathrm{C}=\mathrm{N}), 1219(\mathrm{OMe})$; ${ }^{1} \mathrm{H}-\mathrm{NMR}\left(500 \mathrm{MHz}, \mathrm{DMSO}-\mathrm{d}_{6}, \delta / \mathrm{ppm}\right): 1.45\left(\mathrm{~m}, 2 \mathrm{H}, \mathrm{CH}_{2}\right), 1.50-1.81$ $\left(m, 4 \mathrm{H}, 2 \mathrm{CH}_{2}\right), 1.52\left(t, 2 \mathrm{H}, \mathrm{CH}_{2}\right), 1.65\left(m, 2 \mathrm{H}, \mathrm{CH}_{2}\right), 1.74(m, 2 \mathrm{H}$, $\left.\mathrm{CH}_{2}\right), 1.75-2.20\left(q, 4 \mathrm{H}, 2 \mathrm{CH}_{2}\right), 1.82\left(t, 2 \mathrm{H}, \mathrm{CH}_{2}\right), 2.50(m, 1 \mathrm{H}, \mathrm{CH})$, $3.77\left(s, 3 \mathrm{H}, \mathrm{OCH}_{3}\right), 3.80\left(s, 3 \mathrm{H}, \mathrm{OCH}_{3}\right), 3.83\left(s, 3 \mathrm{H}, \mathrm{OCH}_{3}\right), 4.45(s, 1 \mathrm{H}$, $\mathrm{CH}$, thiazine), $10.98(\mathrm{~s}, 1 \mathrm{H}, \mathrm{NH}), 6.61-7.90(\mathrm{~m}, 15 \mathrm{H}, \mathrm{Ar}-\mathrm{H})$; $\mathrm{MS}(\mathrm{m} / \mathrm{z}$, (relative abundance, \%)): $834\left(\mathrm{M}^{+}, 40\right)$; Anal. Calcd. for $\mathrm{C}_{48} \mathrm{H}_{46} \mathrm{~N}_{6} \mathrm{O}_{6} \mathrm{~S}: \mathrm{C}$, 69.05; H, 5.55; N, 10.06; Found: C, 69.10; H, 5.23; N, 10.02 .

3-(2-(10-cyclohexyl-5-(3,4-dimethoxyphenyl)-4-oxo-3,4,5,6,7,8,9,10octahydro-pyrimido[4,5-b]quinolin-2-yl)-4-oxoquinazolin-3(4H)-yl)-2(4-fluorophenyl)-2H-benzo[e][1,3]thiazin-4(3H)-one (29b)

Yellow crystals; Yield 53\%; m.p. $115-116^{\circ} \mathrm{C}$; IR $\left(\mathrm{KBr}, \mathrm{cm}^{-1}\right): 3396(\mathrm{NH})$, 1740, 1735, $1720(\mathrm{C}=\mathrm{O}), 1690,1650(\mathrm{C}=\mathrm{N}), 1219(\mathrm{OMe}) ;{ }^{1} \mathrm{H}-\mathrm{NMR}$ (500 MHz, DMSO-d 6 , $\delta / \mathrm{ppm}): 1.45\left(m, 2 \mathrm{H}, \mathrm{CH}_{2}\right), 1.50-1.81(m, 4 \mathrm{H}$, $\left.2 \mathrm{CH}_{2}\right), 1.52\left(t, 2 \mathrm{H}, \mathrm{CH}_{2}\right), 1.65\left(m, 2 \mathrm{H}, \mathrm{CH}_{2}\right), 1.74\left(m, 2 \mathrm{H}, \mathrm{CH}_{2}\right), 1.75-$ $2.20\left(q, 4 \mathrm{H}, 2 \mathrm{CH}_{2}\right), 1.82\left(t, 2 \mathrm{H}, \mathrm{CH}_{2}\right), 2.50(m, 1 \mathrm{H}, \mathrm{CH}), 3.78(s, 3 \mathrm{H}$, $\left.\mathrm{OCH}_{3}\right), 3.80\left(s, 3 \mathrm{H}, \mathrm{OCH}_{3}\right), 4.45(s, 1 \mathrm{H}, \mathrm{CH}$, thiazine $), 10.98(s, 1 \mathrm{H}$, $\mathrm{NH}), 6.61-7.90(\mathrm{~m}, 15 \mathrm{H}, \mathrm{Ar}-\mathrm{H})$; MS ( $m / z$, (relative abundance, \%)): 822 $\left(\mathrm{M}^{+}, 40\right)$; Anal. Calcd. for $\mathrm{C}_{47} \mathrm{H}_{43} \mathrm{FN}_{6} \mathrm{O}_{5} \mathrm{~S}$ : C, 68.60; H, 5.27; N, 10.21; Found: C, $68.10 ; \mathrm{H}, 5.23 ; \mathrm{N}, 10.02$.

\section{Biological activity}

\section{Test microorganisms}

Standard strains used to evaluate antimicrobial activity; Gram positive bacteria; (Bacillus subtilis ATCC 6633 and Staphylococcus aureus ATCC 6538-P), Gram negative bacteria (Pseudomonas aeruginosa ATCC 27853 and Bordetella pertussis ATCC 9797), yeasts (Candida albicans ATCC 10231 and Saccharomyces cervesiae) and fungi (Aspergillus niger NRRL A-326 and Trichoderma viride NRC 314) were obtained from culture collection stocks maintained in the Department of Microbial Chemistry, National Research Centre, Egypt. Bacteria were maintained at $4{ }^{\circ} \mathrm{C}$ on nutrient agar slants containing $(\mathrm{g} / \mathrm{L})$ : beef extract, 3; peptone, 5 and $1 \mathrm{~L}$ of distilled water, and adjusted at $\mathrm{pH} 7.2$ before autoclaving (LAC-J0805 autoclave, Daihan Labtech Co., Korea) at $121{ }^{\circ} \mathrm{C}$ for 15 min, while yeast and fungi were maintained on Sabouraud dextrose agar (SDA) and Potato dextrose agar (PDA) media, respectively.

\section{Antimicrobial activity}

The antimicrobial activity of each chemical compound was investigated in vitro by the Department of Microbial Chemistry, National Research Centre using the agar well diffusion method (WDM) recommended by the Clinical and Laboratories Standards Institute (CLSI) to measure in vitro susceptibility of bacteria to antimicrobial agents used in clinical settings. The accuracy of this test depends on the maintenance of standard procedures. In the present study, a stock solution containing $20 \mathrm{mg} / \mathrm{mL}$ in DMSO is prepared for each chemical compound. Dispense nutrient agar seeded with $1.5 \times 10^{8} \mathrm{CFU} / \mathrm{mL}$ of each bacterial strain, SDA seeded with $2.0 \times 10^{5} \mathrm{CFU} / \mathrm{mL}$ of each yeast and PDA seeded with $2.0 \times 10^{4} \mathrm{CFU} / \mathrm{mL}$ for each fungal strain (cooled below $45^{\circ} \mathrm{C}$ ) into sterile Petri dishes, give a depth of $4 \mathrm{~mm}(\sim 20 \mathrm{~mL}$ in Petri dish of 85 $\mathrm{mm}$ in diameter). Allow the agar to set before moving the plates. Agar 
wells of diameter $8 \mathrm{~mm}$ were made in the agar plates with the help of a sterilized cork borer. Wells were loaded with $100 \mu \mathrm{L}(20 \mathrm{mg} / \mathrm{mL})$ of tested compound solutions and controls under aseptic condition. These plates were sealed with parafilm and kept in the refrigerator for $4 \mathrm{~h}$ at 5 ${ }^{\circ} \mathrm{C}$ for the complete diffusion of antimicrobial compounds, if any.

Thereafter, the sealed plates were incubated upright at $35^{\circ} \mathrm{C}$ for 18 $24 \mathrm{~h}$ for bacteria and yeasts, and $48-72 \mathrm{~h}$ at $28^{\circ} \mathrm{C}$ for fungi. Positive control experiments were conducted under similar conditions using cefaxone $(20 \mathrm{mg} / \mathrm{mL})$, Ketoconazole $(20 \mathrm{mg} / \mathrm{mL})$ and cyclosporine $(10 \mathrm{mg} / \mathrm{mL})$ as standard drugs for antibacterial and antifungal activity, respectively. Similarly, $10 \mu \mathrm{L}$ DMSO was used as a negative control. After the incubation period, antimicrobial activity was evaluated by measuring the diameter of inhibition zone in millimeters $(\mathrm{mm})$ and compared to that of the standard (Positive controls). Inhibition zones with a diameter $\geq 16 \mathrm{~mm}$ were considered to have antimicrobial activity for further quantitative tests of their activity. The experiment was performed in triplicate and the average inhibition zone was calculated.

\section{Determination of minimal inhibitory concentration} (MIC)

In microbiology, the minimum inhibitory concentration (MIC) endpoints were defined as the lowest concentration of the assayed antimicrobial agent, which resulted in a $100 \%$ reduction in growth compared to the antimicrobial agent-free growth control test ${ }^{23}$. The bacteriostatic activity of the active chemical compounds (with inhibition zones $\geq 16 \mathrm{~mm}$ ) was evaluated using a two-fold serial dilution technique $^{24}$. Two-fold serial dilutions of the tested compound solutions were prepared using the proper nutrient broth. The final concentrations of the solutions were 25, 50, 75, 100, 150, 200 and $300 \mu \mathrm{g} / \mathrm{mL}$. Each 5.0 $\mathrm{mL}$ received $0.1 \mathrm{~mL}$ of inoculums and incubated at $37^{\circ} \mathrm{C}$ for $24 \mathrm{~h}$ for bacteria and yeasts, and $48 \mathrm{~h}$ at $28^{\circ} \mathrm{C}$ for fungi. Tests were performed in triplicate and repeated twice. The lowest concentration showing no growth was considered the minimum inhibitory concentration (MIC) (Table 3).

\section{Antioxidant activity of chemical compounds}

\section{Evaluation of antioxidant activity using the DPPH radical scavenging method}

The percentage of antioxidant activity of each chemical compound was measured by the Department of Microbial Chemistry, National Research Centre using the 1,1-Diphenyl-2-picrylhydrazyl (DPPH) radical scavenging assay ${ }^{25}$. This assay is based on the measurement of the ability of antioxidants to reduce DPPH by measuring the decrease in its absorption ${ }^{26}$. DPPH reacts with hydrogen/electron donor compounds and has a maximum UV-Vis absorption of 515$520 \mathrm{~nm}^{27}$. The reaction mixture consisted of $50 \mu \mathrm{L}(10-200 \mu \mathrm{g} / \mathrm{mL})$ of each chemical compound dissolved in dimethyl sulfoxide (DMSO), as well as the reference standard ascorbic acid and the volume was made uniformly to $150 \mu \mathrm{L}$ using ethanol, $3 \mathrm{~mL}$ of absolute ethanol and 150 $\mu \mathrm{L}$ of freshly prepared DPPH radical solution $(0.5 \mathrm{mM}$ in ethanol). The mixtures were shaken vigorously and left to stand in the dark for $30 \mathrm{~min}$ at room temperature, and the absorbance was measured at $517 \mathrm{~nm}$ in Cary-100 UV-Vis spectrophotometer (Agilent Technologies, Frankfurt, Germany) using ethanol as a blank. Control reactions were performed without the test sample (i.e. $150 \mu \mathrm{L}$ of $\mathrm{DPPH}+3.0 \mathrm{~mL}$ ethanol). The experiment was carried out in triplicate for each chemical compound. Radical scavenging capacity was expressed as a percentage (\%) and was calculated using the following formula:

Radical scavenging activity $(\%)=\frac{\left(\mathrm{Abs}_{\text {control }}-\mathrm{Abs}_{\text {sample }}\right) \times 100}{\mathrm{Abs} \mathrm{control}}$
$\mathrm{Abs}_{\text {control }}$ and $\mathrm{Abs}$ sample : the absorbance values of the control as well as the sample.

The antioxidant activity of each chemical compound and ascorbic acid was expressed as $\mathrm{EC}_{50}$ (the effective micromolar concentration required to scavenge $50 \%$ of DPPH radicals) is a typically employed parameter to express the antioxidant capacity and to compare the activity of different compounds ${ }^{28}$ (Table 1). It is worth note that EDTA was added to prevent ascorbic acid oxidation.

\section{RESULTS AND DISCUSSION}

\section{Chemistry}

Compound 1 was synthesized previously by the author ${ }^{29}$. In scheme 1 , anthranilic acid (2) reacted with excess of acid chloride derivatives (1) in presence of dry pyridine to afford quinolin-oxazin-4-one derivative ${ }^{30}$ (3). IR spectrum of compound 3 demonstrated two bands of $\mathrm{C}=\mathrm{O}$ and $\mathrm{NH}$ at $\approx 1715,1745$ and $3450 \mathrm{~cm}^{-1}$, respectively (Scheme 1 ). ${ }^{1} \mathrm{H}$-NMR of 3 showed singlet tow $\mathrm{OCH}_{3}$ and $\mathrm{NH}$ signals occurring at 3.72, 3.80 and $10.5 \mathrm{ppm}$, respectively. Compound 3 play as electrophilic intermediate key for the synthesis of interest pharmaceutical derivatives.

In scheme 2, also, the compound 3 refluxed with compound 4 in absolute EtOH to afford carboxamide compounds (5). IR of compound 5 revealed 3 groups $\mathrm{C}=\mathrm{O}$ at $\approx 1750,1740,1725$ and two $\mathrm{NH}_{2}$ and only one NH. (Scheme 2). ${ }^{1} \mathrm{H}-\mathrm{NMR}$ of 5 revealed $\mathrm{NH}_{2}$ at $5.5 \mathrm{ppm}$. Moreover, compound 3 refluxed with $\mathrm{CH}_{3} \mathrm{COONH}_{4}$ afford quinazolin-4(3H)-one (6). Moreover, in dry xylene/toluene compound 3 refluxed with $\mathrm{P}_{2} \mathrm{~S}_{5}$ to afford thiazin-4-one (7). Where elemental analysis of 7 revealed $S$ $4.48 \%$.

In scheme 3, compound 3 allowed to react with a series of primary heterocyclic amines and hydrazine hydrate $(8 \mathrm{a}-\mathrm{d})$ to afford $9 \mathrm{a}-\mathrm{d}$ and fused with $2 / 3$-aminopyridine $(10,12)$ in presence of $\mathrm{ZnCl}_{2}$ to yielded 11,13 , respectively. Also, compound 3 refluxed with $\mathrm{NH}_{2} \mathrm{OH} . \mathrm{HCl}$ in presence of $\mathrm{CH}_{3} \mathrm{COONa}$ to afford 3-hydroxyquinazolin-4-one (15). IR of compound 15 revealed the presence of $-\mathrm{OH}$ group at $\approx 3635 \mathrm{~cm}^{-1}$, where ${ }^{1} \mathrm{H}-\mathrm{NMR}$ of 15 proved the presence of $\mathrm{OH}$ at $6.1 \mathrm{ppm}$ (Scheme 3).

In scheme 4, The nucleophilic amino group of compound 9a condensed with series of aldehydes (16a-d) in presence of piperidine to afford benzylidene-quinazoline (17a-d). Also, 9a refluxed in dry pyridine with $\mathrm{CH}_{3} \mathrm{COCl}$ or phCOCl to afford 19, 21a, b, respectively (Scheme 4). In addition to, 9a fused with succinic anhydride and phthalic anhydride (22a, b) to yield the corresponding compounds 23a, b.

In last scheme 5 , hexahydropyrimido[4,5-b]quinolin was prepared by refluxing of compound $17 \mathrm{a}, \mathrm{b}$ with $\mathrm{CH}_{2} \mathrm{COCl}_{2}$ in $\mathrm{EtOH}$ in presence of $\mathrm{Et}_{3} \mathrm{~N}$ to afford compounds $25 \mathrm{a}$, b. In addition to, compound $17 \mathrm{a}, \mathrm{b}$ refluxed with thioglycolic acid or thiosalicylic acid in dry benzene to yield new thiazolidin-4-one 27a, b and thiazin-one 29a, b, respectively.

\section{Biological activity}

\section{In vitro antimicrobial Screening}

The newly synthesized compounds were evaluated as antimicrobial agents. It was observed that from Table 1 and 2, the compound $17 \mathrm{~b}$ produced the most potent inhibitory activity against the growth of the strains tested. The MIC of compound $17 \mathrm{~b}$ was equivalent to that of all standard drugs used $(25-50 \mu \mathrm{g} / \mathrm{mL})$. Interestingly, the compound $17 \mathrm{~b}$ produced potent antifungal activity against Trichoderma viride that is greater than the Cyclosporine reference drug (MIC Cyclosporine; $50 \mu \mathrm{g} / \mathrm{mL}$, MIC compound $17 \mathrm{~b} ; 25 \mu \mathrm{g} / \mathrm{mL}$ ). 
Table 1: Antimicrobial activity of based on well diffusion method (100 $\boldsymbol{\mu L}=\mathbf{2 0 0 0} \boldsymbol{\mu g}$ ).

\begin{tabular}{|c|c|c|c|c|c|c|c|c|}
\hline Compound & $\begin{array}{c}\text { Staphylococcus } \\
\text { aureus } \\
\text { ATCC 6538-P }\end{array}$ & $\begin{array}{l}\text { Bacillus subtilis } \\
\text { ATCC } 6633\end{array}$ & $\begin{array}{c}\text { Pseudomonas } \\
\text { aeruginosa } \\
\text { ATCC } 27853\end{array}$ & $\begin{array}{l}\text { Bordetella } \\
\text { pertussis } \\
\text { ATCC- } 9797\end{array}$ & $\begin{array}{c}\text { Candida } \\
\text { albicans ATCC- } \\
10231\end{array}$ & $\begin{array}{c}\text { Saccharomyces } \\
\text { cervesiae }\end{array}$ & $\begin{array}{c}\text { Aspergillus } \\
\text { niger } \\
\text { NRRL } \\
\text { A-326 }\end{array}$ & $\begin{array}{c}\text { Trichoderma } \\
\text { viride } \\
\text { NRC } 314\end{array}$ \\
\hline 3 & $\mathrm{R}$ & $\mathrm{R}$ & $\mathrm{R}$ & $\mathrm{R}$ & $\mathrm{R}$ & $\mathrm{R}$ & $\mathrm{R}$ & $\mathrm{R}$ \\
\hline 5 & $\mathrm{R}$ & $\mathrm{R}$ & $\mathrm{R}$ & $\mathrm{R}$ & $\mathrm{R}$ & $\mathrm{R}$ & $\mathrm{R}$ & $\mathrm{R}$ \\
\hline 6 & $\mathrm{R}$ & $\mathrm{R}$ & $\mathrm{R}$ & $\mathrm{R}$ & 20 & 18 & $\mathrm{R}$ & $\mathrm{R}$ \\
\hline 7 & $\mathrm{R}$ & $\mathrm{R}$ & $\mathrm{R}$ & 13 & 14 & $\mathrm{R}$ & $\mathrm{R}$ & $\mathrm{R}$ \\
\hline $9 a$ & $\mathrm{R}$ & $\mathrm{R}$ & $\mathrm{R}$ & $\mathrm{R}$ & 21 & 20 & $\mathrm{R}$ & $\mathrm{R}$ \\
\hline $9 b$ & $\mathrm{R}$ & $\mathrm{R}$ & $\mathrm{R}$ & $\mathrm{R}$ & 22 & 25 & $\mathrm{R}$ & $\mathrm{R}$ \\
\hline $9 c$ & 23 & 25 & 18 & 16 & 25 & 26 & $\mathrm{R}$ & $\mathrm{R}$ \\
\hline $9 d$ & 31 & 33 & 32 & 30 & 27 & 30 & 28 & 31 \\
\hline 11 & $\mathrm{R}$ & $\mathrm{R}$ & $\mathrm{R}$ & $\mathrm{R}$ & $\mathrm{R}$ & $\mathrm{R}$ & $\mathrm{R}$ & $\mathrm{R}$ \\
\hline 13 & $\mathrm{R}$ & $\mathrm{R}$ & $\mathrm{R}$ & 12 & 25 & 20 & $\mathrm{R}$ & $\mathrm{R}$ \\
\hline 15 & $\mathrm{R}$ & 16 & 22 & 25 & 27 & 25 & 24 & 30 \\
\hline $17 \mathrm{a}$ & $\mathrm{R}$ & $\mathrm{R}$ & $\mathrm{R}$ & $\mathrm{R}$ & 21 & 20 & $\mathrm{R}$ & $\mathrm{R}$ \\
\hline $17 \mathrm{~b}$ & 36 & 35 & 33 & 37 & 29 & 33 & 34 & 35 \\
\hline $17 \mathrm{c}$ & 23 & 30 & 14 & 16 & 20 & 16 & $\mathrm{R}$ & $\mathrm{R}$ \\
\hline $17 \mathrm{~d}$ & $\mathrm{R}$ & $\mathrm{R}$ & $\mathrm{R}$ & $\mathrm{R}$ & $\mathrm{R}$ & $\mathrm{R}$ & $\mathrm{R}$ & $\mathrm{R}$ \\
\hline 19 & 20 & 23 & 16 & 18 & 20 & 17 & $\mathrm{R}$ & $\mathrm{R}$ \\
\hline $21 \mathrm{a}$ & $\mathrm{R}$ & $\mathrm{R}$ & $\mathrm{R}$ & $\mathrm{R}$ & 15 & 12 & $\mathrm{R}$ & $\mathrm{R}$ \\
\hline $21 b$ & $\mathrm{R}$ & 12 & $\mathrm{R}$ & $\mathrm{R}$ & 13 & $\mathrm{R}$ & $\mathrm{R}$ & $\mathrm{R}$ \\
\hline $23 a$ & 11 & 15 & 22 & 24 & 20 & 20 & $\mathrm{R}$ & $\mathrm{R}$ \\
\hline $23 b$ & $\mathrm{R}$ & $\mathrm{R}$ & 14 & 15 & 22 & 20 & $\mathrm{R}$ & $\mathrm{R}$ \\
\hline $25 a$ & $\mathrm{R}$ & $\mathrm{R}$ & $\mathrm{R}$ & $\mathrm{R}$ & 20 & 17 & $\mathrm{R}$ & $\mathrm{R}$ \\
\hline $25 b$ & $\mathrm{R}$ & 12 & 18 & 20 & 22 & 20 & $\mathrm{R}$ & $\mathrm{R}$ \\
\hline $27 \mathrm{a}$ & $\mathrm{R}$ & $\mathrm{R}$ & $\mathrm{R}$ & $\mathrm{R}$ & 20 & $\mathrm{R}$ & $\mathrm{R}$ & $\mathrm{R}$ \\
\hline $27 \mathrm{~b}$ & $\mathrm{R}$ & $\mathrm{R}$ & $\mathrm{R}$ & 12 & 20 & 16 & $\mathrm{R}$ & $\mathrm{R}$ \\
\hline $29 a$ & 12 & 13 & 12 & 15 & 24 & 20 & $\mathrm{R}$ & $\mathrm{R}$ \\
\hline $29 b$ & 16 & 14 & 17 & 19 & 31 & 26 & $\mathrm{R}$ & $\mathrm{R}$ \\
\hline Negative Control & $\mathrm{R}$ & $\mathrm{R}$ & $\mathrm{R}$ & $\mathrm{R}$ & $\mathrm{R}$ & $\mathrm{R}$ & $\mathrm{R}$ & $\mathrm{R}$ \\
\hline Cefaxone & 38 & 36 & 34 & 39 & NT & NT & NT & NT \\
\hline Ketoconazole & NT & NT & NT & NT & 31 & 34 & NT & NT \\
\hline Cyclosporine & NT & NT & NT & NT & NT & NT & 35 & 32 \\
\hline
\end{tabular}

$\mathrm{R}=$ Resistant. NT $=$ Not tested.

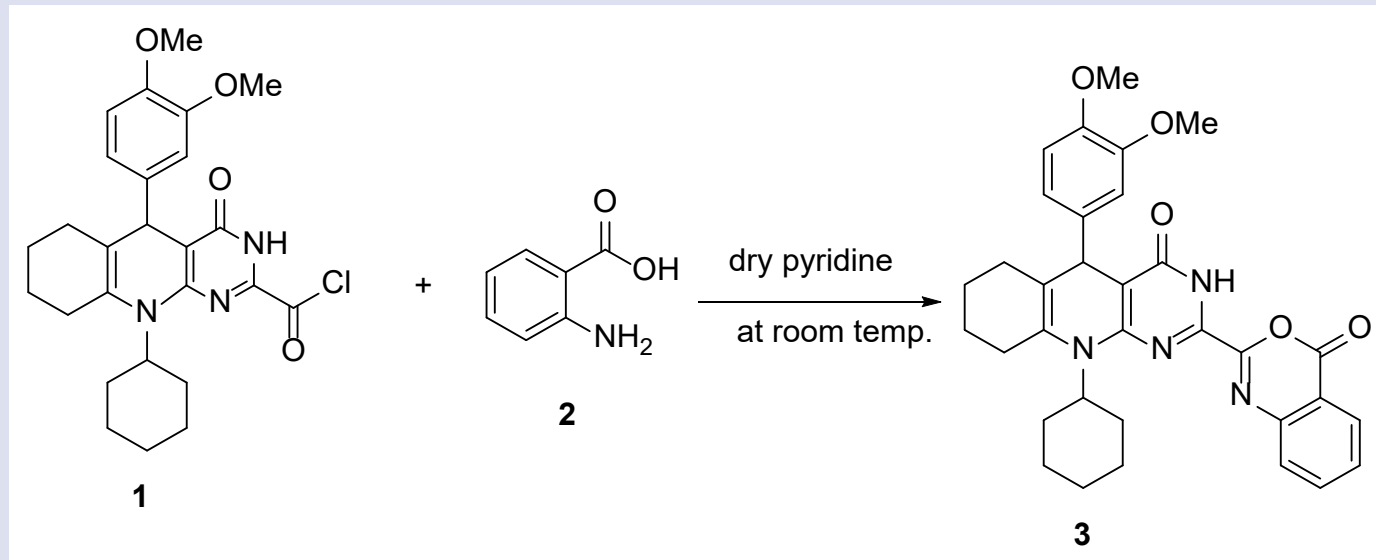

Scheme 1: Synthesis of quinoline-oxazin-4-one derivative. 

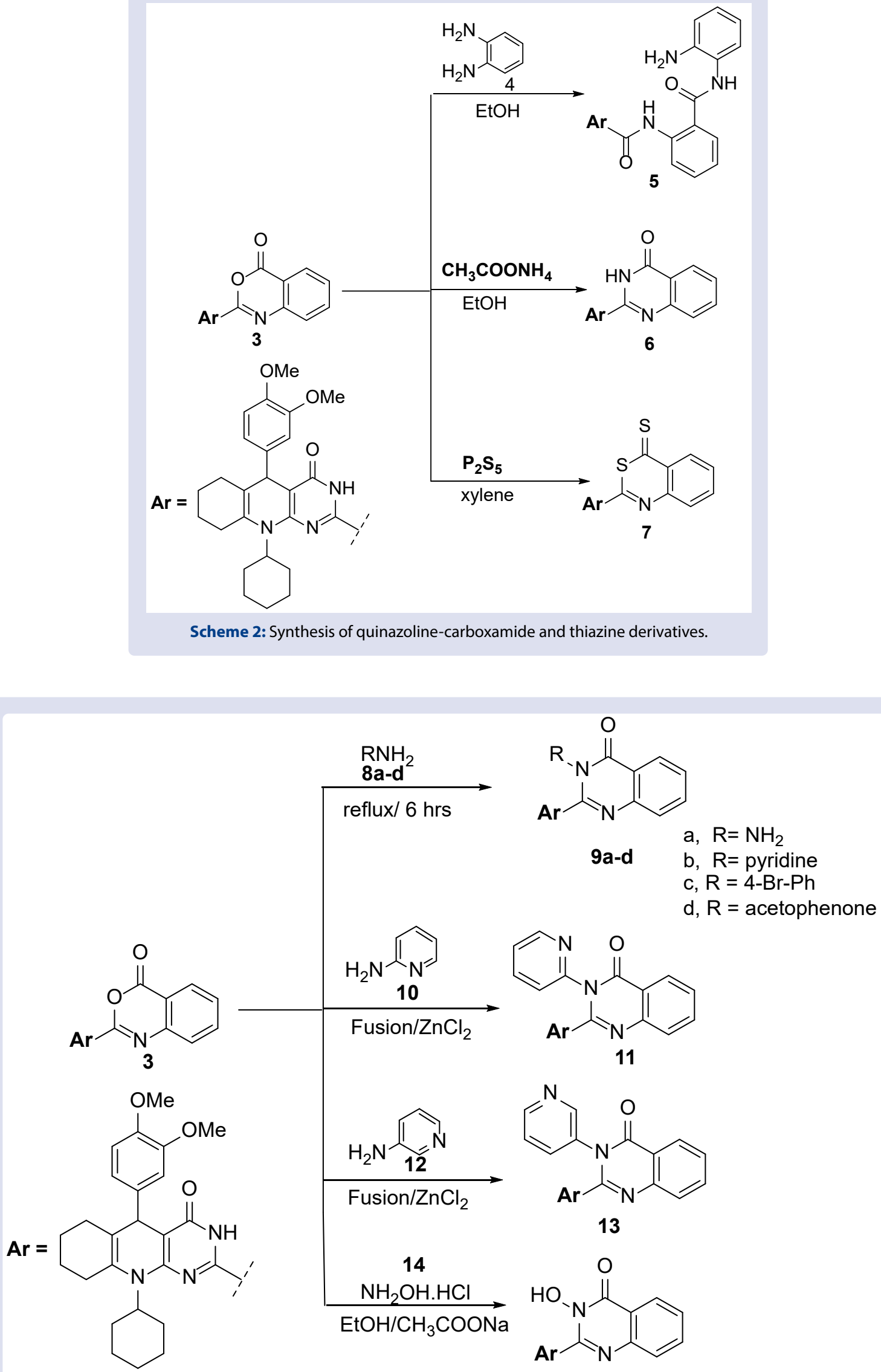


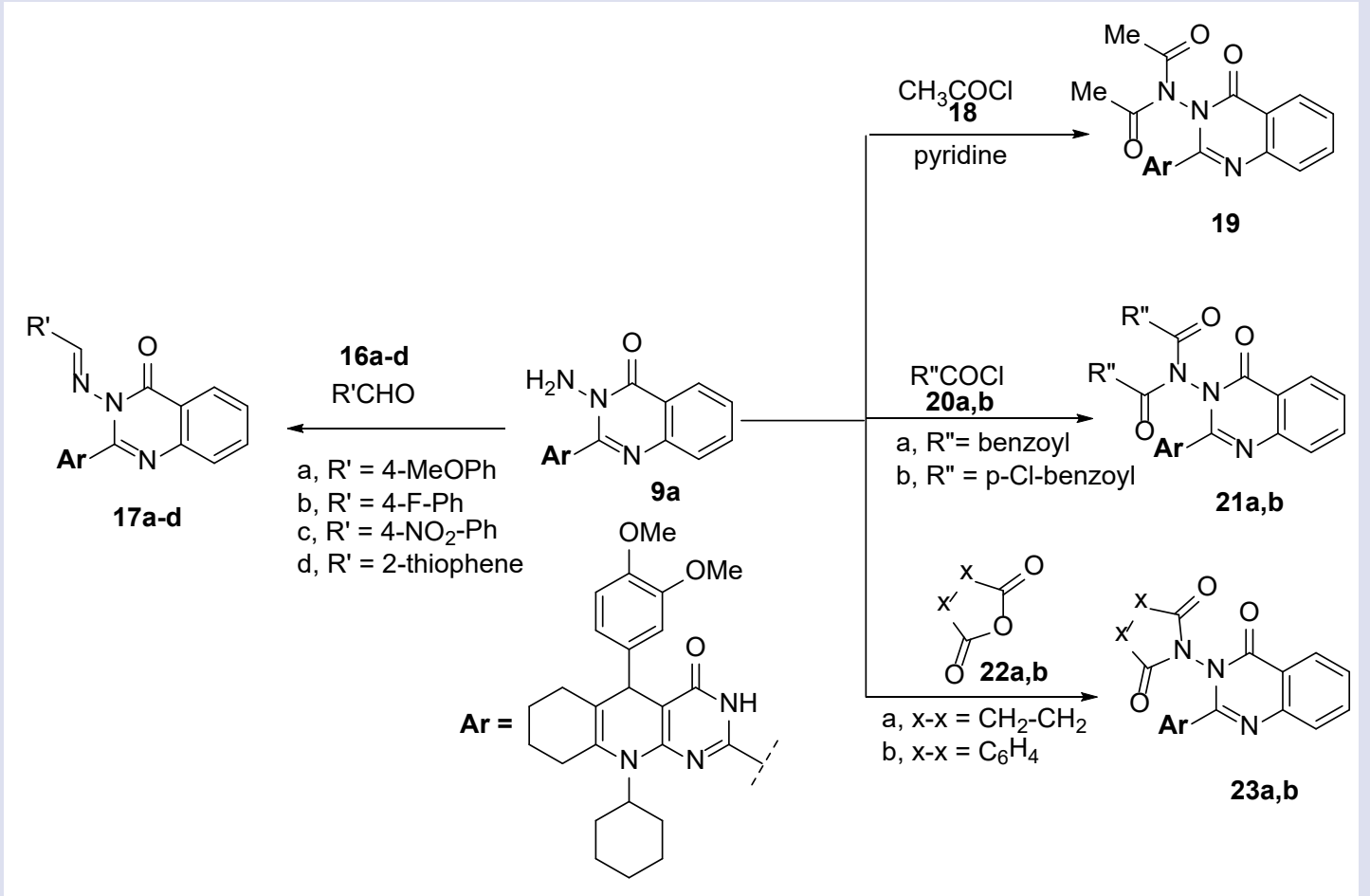

Scheme 4: Synthesis of 4-oxoquinazolin derivatives.

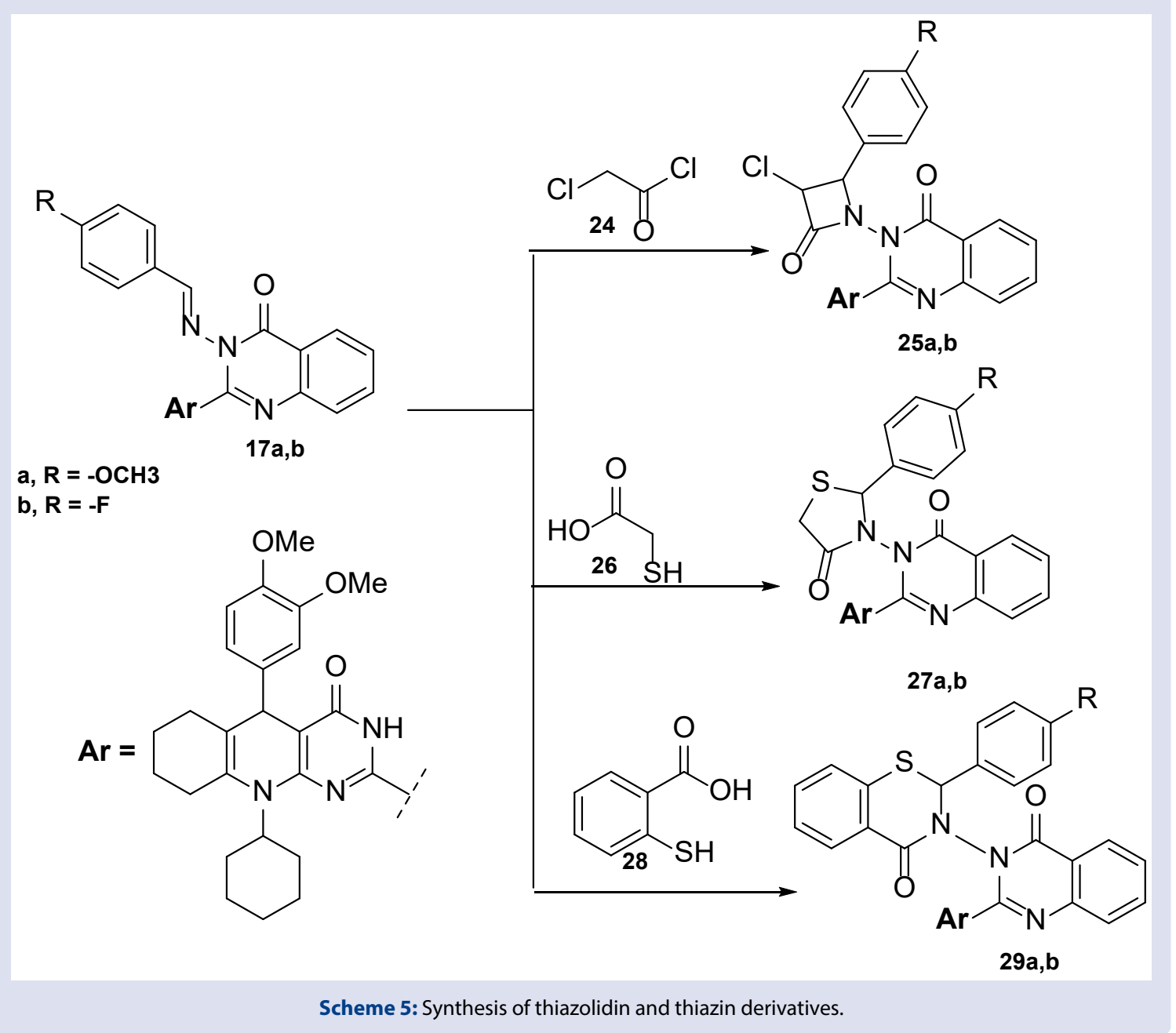


Table 2: MIC $(\mu \mathrm{g} / \mathrm{mL})$ against the pathological strains based on two folds serial dilution technique.

\begin{tabular}{|c|c|c|c|c|c|c|c|c|}
\hline Compound & $\begin{array}{c}\text { Staphylococcus } \\
\text { aureus } \\
\text { ATCC 6538-P }\end{array}$ & $\begin{array}{c}\text { Bacillus subtilis } \\
\text { ATCC } 6633\end{array}$ & $\begin{array}{c}\text { Pseudomonas } \\
\text { aeruginosa } \\
\text { ATCC } 27853\end{array}$ & $\begin{array}{l}\text { Bordetella } \\
\text { pertussis } \\
\text { ATCC } 9797\end{array}$ & $\begin{array}{c}\text { Candida } \\
\text { albicans ATCC } \\
10231\end{array}$ & $\begin{array}{c}\text { Saccharomyces } \\
\text { cervesiae }\end{array}$ & $\begin{array}{c}\text { Aspergillus } \\
\text { niger } \\
\text { NRRL } \\
\text { A-326 }\end{array}$ & $\begin{array}{c}\text { Trichoderma } \\
\text { viride } \\
\text { NRC } 314\end{array}$ \\
\hline 3 & NT & NT & NT & NT & NT & NT & NT & NT \\
\hline 5 & NT & NT & NT & NT & NT & NT & NT & NT \\
\hline 6 & NT & NT & NT & NT & 100 & 150 & NT & NT \\
\hline 7 & NT & NT & NT & NT & NT & NT & NT & NT \\
\hline $9 a$ & NT & NT & NT & NT & 75 & 100 & NT & NT \\
\hline $9 b$ & NT & NT & 200 & NT & NT & 50 & NT & NT \\
\hline $9 c$ & 75 & 75 & 200 & 300 & 75 & 50 & NT & NT \\
\hline $9 d$ & 50 & 50 & 50 & 75 & 50 & 50 & 75 & 50 \\
\hline 11 & NT & NT & NT & NT & NT & NT & NT & NT \\
\hline 13 & NT & NT & NT & NT & 75 & 100 & NT & NT \\
\hline 15 & NT & 300 & 100 & 75 & 50 & 75 & 75 & 50 \\
\hline $17 \mathrm{a}$ & NT & NT & NT & NT & 100 & 150 & NT & NT \\
\hline $17 \mathrm{~b}$ & 50 & 25 & 25 & 50 & 50 & 25 & 25 & 25 \\
\hline $17 \mathrm{c}$ & NT & NT & NT & NT & NT & NT & NT & NT \\
\hline $17 d$ & NT & NT & NT & NT & NT & NT & NT & NT \\
\hline 19 & 100 & 75 & 300 & 200 & 150 & 200 & NT & NT \\
\hline $21 \mathrm{a}$ & NT & NT & NT & NT & NT & NT & NT & NT \\
\hline $21 b$ & NT & NT & NT & NT & NT & NT & NT & NT \\
\hline $23 a$ & NT & NT & 100 & 75 & 200 & 200 & NT & NT \\
\hline $23 b$ & NT & NT & NT & NT & 100 & 200 & NT & NT \\
\hline $25 a$ & NT & NT & NT & NT & 200 & 300 & NT & NT \\
\hline $25 b$ & NT & NT & 200 & 100 & 100 & 200 & NT & NT \\
\hline $27 \mathrm{a}$ & NT & NT & NT & NT & 200 & NT & NT & NT \\
\hline $27 \mathrm{~b}$ & NT & NT & NT & NT & 200 & 300 & NT & NT \\
\hline $29 \mathrm{a}$ & NT & NT & NT & NT & 75 & 200 & NT & NT \\
\hline $29 b$ & 300 & NT & 300 & 200 & 50 & 75 & NT & NT \\
\hline Cefaxone & 25 & 25 & 25 & 25 & NT & NT & NT & NT \\
\hline Ketoconazole & NT & NT & NT & NT & 50 & 25 & NT & NT \\
\hline Cyclosporine & NT & NT & NT & NT & NT & NT & 25 & 50 \\
\hline
\end{tabular}

NT $=$ Not tested.

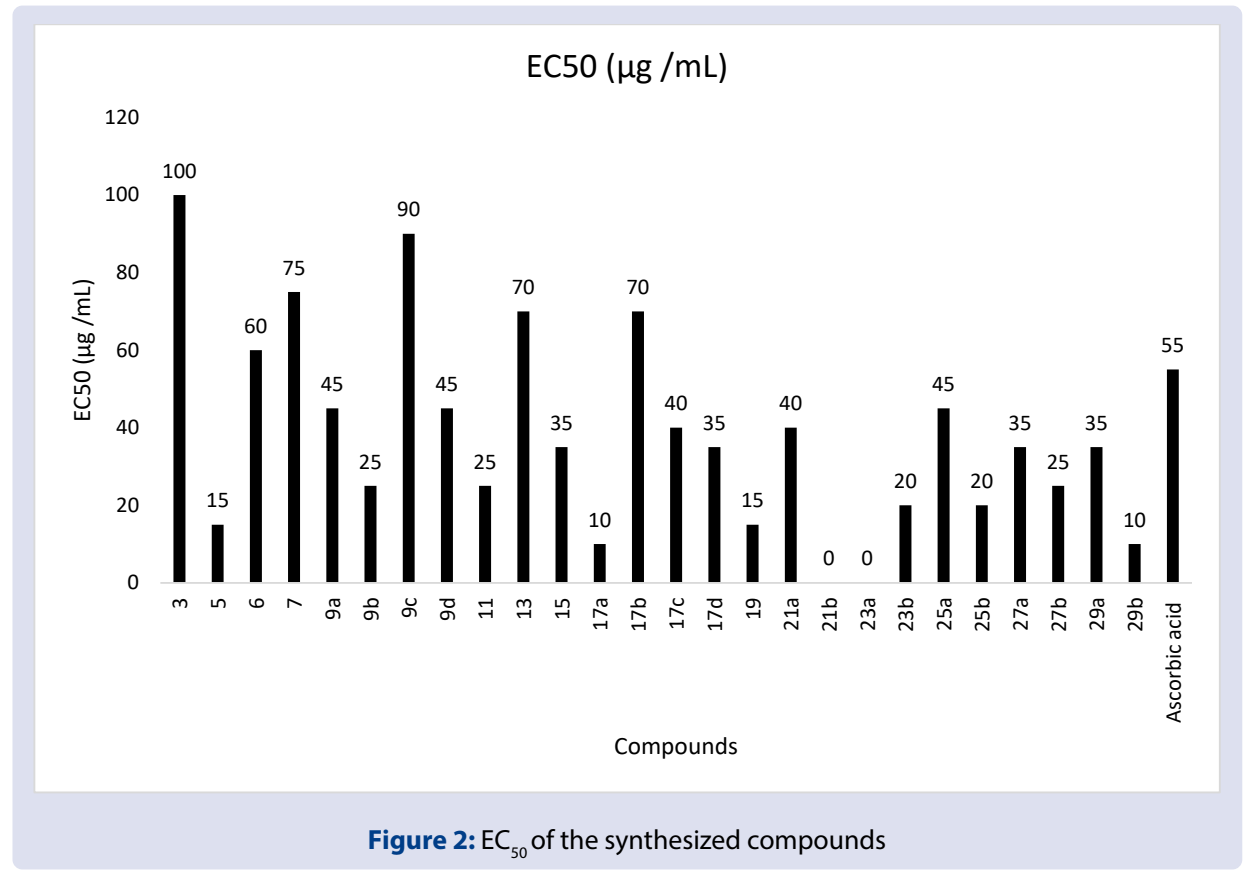


Table 3: $\mathrm{EC}_{50}$ for DPPH inhibition of chemical compounds.

\begin{tabular}{cc}
\hline Chemical compound & $\mathrm{EC}_{50}(\mu \mathrm{g} / \mathrm{mL})$ \\
\hline 3 & 100 \\
5 & 15 \\
6 & 60 \\
7 & 75 \\
$9 \mathrm{a}$ & 45 \\
$9 \mathrm{~b}$ & 25 \\
$9 \mathrm{c}$ & 90 \\
$9 \mathrm{~d}$ & 45 \\
11 & 25 \\
13 & 70 \\
15 & 35 \\
$17 \mathrm{a}$ & 10 \\
$17 \mathrm{~b}$ & 70 \\
$17 \mathrm{c}$ & 40 \\
$17 \mathrm{~d}$ & 35 \\
19 & 15 \\
$21 \mathrm{a}$ & 40 \\
$21 \mathrm{~b}$ & $\mathrm{NA}$ \\
$23 \mathrm{a}$ & $\mathrm{NA}$ \\
$23 \mathrm{~b}$ & 20 \\
$25 \mathrm{a}$ & 45 \\
$25 \mathrm{~b}$ & 20 \\
$27 \mathrm{a}$ & 35 \\
$27 \mathrm{~b}$ & 25 \\
$29 \mathrm{a}$ & 35 \\
$29 \mathrm{~b}$ & 10 \\
Ascorbic acid (Control) & 55 \\
\hline &
\end{tabular}

$\mathrm{NA}=$ Not active.

\section{Antioxidant activity}

The compounds were evaluated as antioxidant agents and compared with reference drug (ascorbic acid) (Table 3 ). The obtained potency was as follows: $17 \mathrm{a}=29 \mathrm{~b}=10 \mu \mathrm{g} / \mathrm{mL}>5=19=15 \mu \mathrm{g} / \mathrm{mL}>23 \mathrm{~b}=$ $25 \mathrm{~b}=20 \mu \mathrm{g} / \mathrm{mL}>9 \mathrm{~b}=11=27 \mathrm{~b}=25 \mu \mathrm{g} / \mathrm{mL}>15=17 \mathrm{~d}=27 \mathrm{a}=29 \mathrm{a}$ $=35 \mu \mathrm{g} / \mathrm{mL}>17 \mathrm{c}=21 \mathrm{a}=40 \mu \mathrm{g} / \mathrm{mL}>9 \mathrm{a}=9 \mathrm{~d}=25 \mathrm{a}=45 \mu \mathrm{g} / \mathrm{mL}>$ Ascorbic acid $\left(\mathrm{EC}_{50} ; 55 \mu \mathrm{g} / \mathrm{mL}\right)>6=60 \mu \mathrm{g} / \mathrm{mL}>13=17 \mathrm{~b}\left(\mathrm{EC}_{50} ; 70 \mu \mathrm{g} /\right.$ $\mathrm{mL})$. The remaining derivative compounds $(7,8 \mathrm{c}, 3,21 \mathrm{~b}, 23 \mathrm{a})$ exhibited moderate to non-antioxidant activity.

\section{CONCLUSION}

A new series of pyrimido[4,5-b] quinoline and benzoxazinones derivatives were synthesized and tested to antioxidant and antimicrobial activity. Results revealed that some of these novel compounds displayed significant biological activity. The compounds $17 \mathrm{~b}, 9 \mathrm{~d}$ and $9 \mathrm{c}$, showed high promising antimicrobial activity along with several compounds, in addition to, the compounds $17 \mathrm{a}=29 \mathrm{~b}=10 \mu \mathrm{g} / \mathrm{mL}$ showed the most potent antioxidant agents than ascorbic acid.

In the study of the relationship SARs, very good antimicrobial activity was found at the compounds pyrimido[4,5-b] quinoline derivatives (17b, 9d and 9c) against the test microorganisms. Also, pyrimido[4,5-b]quinoline derivatives and oxoquinazolin-benzo $[1,3]$ thiazin $(17 \mathrm{a}=29 \mathrm{~b}=10 \mu \mathrm{g} / \mathrm{mL})$ possess high antioxidant than agents than ascorbic acid.

\section{REFERENCES}

1. Bawa S, Kumar S, Drabu S, Kumar R. Structural modifications of quinoline-based antimalarial agents: recent developments. J. Pharm. Bioallied Sci. 2010;2:64-71.
2. Teng P, Li C, Peng Z, Marie VA, Nimmagadda A, Su M, et al. Facilely accessible quinoline derivatives as potent antibacterial agents, Bioorg. Med. Chem. 2018;26:3573-9.

3. Keri RS, Patil SA, Quinoline: a promising antitubercular target, Biomed. Pharmacother, 2014;68:1161-75.

4. Fang YM, Zhang RR, Shen ZH, Wu HK, Tan CX, Weng JQ, et al. Synthesis, antifungal activity, and SAR study of some new 6 -perfluoropropanyl quinoline derivatives, J. Heterocycl. Chem. 2018:55:240-5

5. Liang $X$, Wu Q, Luan $S$, Yin Z, He C, Yin L, et al. A comprehensive review of topoisomerase inhibitors as anticancer agents in the past decade, Eur. J. Med. Chem. 2019;171:129-68.

6. Upadhyay A, Kushwaha P, Gupta S, Dodda RP, Ramalingam K, Kant R, et al. Synthesis and evaluation of novel triazolyl quinoline derivatives as potential antileishmanial agents. Eur. J. Med. Chem. 2018;154:172 81.

7. Chaaban I, Rizk OH, Ibrahim TM, Henen SS, El-Khawass ES, Bayad $A E$, et al. Synthesis, anti-inflammatory screening, molecular docking, and COX-1,2/-5-LOX inhibition profile of some novel quinoline derivatives, Bioorg. Chem. 2018;78:220-35.

8. Sharma A, Gupta VK, Pathania R. Efflux pump inhibitors for bacterial pathogens: From bench to bedside. Indian J. Med. Res. 2019;149:12945.

9. Puskullu MO, Celik I, Erol M, Fatullayev H, Uzunhisarcikli E, Kuyucuklu G. Antimicrobial and antiproliferative activity studies of some new quinoline-3-carbaldehyde hydrazone derivatives. Bioorg. Chem. 2020;101:104014-24

10. Katariya KD, Shah SR, Reddy D. Anticancer, antimicrobial activities of quinoline based hydrazone analogues: Synthesis, characterization and molecular docking. Bioorganic Chemistry. 2010;94:103406-19.

11. Aly RM, Serya RA, El-Motwally AM, Esmat A, Abbas S, Abou El Ella DA. Novel quinoline-3-carboxamides (Part 2): Design, optimization and synthesis of quinoline based scaffold as EGFR inhibitors with potent anticancer activity. Bioorg. Chem. 2017;75:368-92.

12. Shao J, Zhu M, Gao L, Chen H, Li X. Synthesis of tetracyclic azasugars fused benzo[e][1,3]thiazin-4-one by the tandem Staudinger/azaWittig/cyclization and their HIV-RT inhibitory activity. Carbohydrate Research. 2018;456:45-52.

13. Yin Z, Zhu M, Wei S, Shao J, Hou Y, Chen H, Li X. Synthesis of tetracyclic iminosugars fused benzo[e][1,3]thiazin-4-one and their HIVRT inhibitory activity. Bioorg. and Med. Chem. Lett. 2016;26:1738-41.

14. El-Gazzar AR, El-Enany MM, Mahmoud MN. Synthesis, analgesic, antiinflammatory, and antimicrobial activity of some novel pyrimido[4,5-b] quinolin-4-ones. Bioorg. and Med. Chem. 2008;16:3261-73.

15. Metwally K, Pratsinis H, Kletsas D. Pyrimido[4,5-c]quinolin-1(2H)ones as a novel class of antimitotic agents: Synthesis and in vitro cytotoxic activity. Euro. J. of Med. Chem. 2007;42:344-50.

16. Hussain F, Khan Z, Jan MS, Ahmad S, Ahmad A, Rashid U, et al. Synthesis, in-vitro $\alpha$-glucosidase inhibition, antioxidant, in-vivo antidiabetic and molecular docking studies of pyrrolidine-2,5-dione and thiazolidine-2,4-dione derivatives. Bioorg. Chem. 2019;91:103128-9.

17. Rapacz A, Rybka S, Obniska J, Jodłowska A, Góra M, Koczurkiewicz $P$, et al. Analgesic and antiallodynic activity of novel anticonvulsant agents derived from 3-benzhydryl-pyrrolidine-2,5-dione in mouse models of nociceptive and neuropathic pain. Euro. J. of Pharma. 2020;869:172890-7.

18. Shariat M, Abdollahi S., Synthesis of benzoxazinone derivatives: a new route to 2-(N-phthaloylmethyl)-4H-3,1-benzoxazin-4-one, Molecules, 2004;9:705-12.

19. Hsieh PW, Chang FR, Chang $\mathrm{CH}$, Cheng PW, Chiang LC, Zeng FL, et al. 2-substituted benzoxazinone analogues as anti-human coronavirus (anti-HCoV) and ICAM-1 expression inhibition agents, Bioorg. Med. Chem. Lett., 2004;14:4751-4.

20. Bari A, Khan ZA, Shahzad SA, Naqvi SA, Khan SA, Amjad H, et al Design and syntheses of 7-nitro-2-aryl-4H-benzo[d][1,3]oxazin-4ones as potent anticancer and antioxidant agents. J. of Molecular Structure. 2020;1214:128252-62. 
21. Marasini BP, Rahim F, Perveen S, Karim A, Khan KM, Choudhary MI. Synthesis, structure-activity relationships studies of benzoxazinone derivatives as a-chymotrypsin inhibitors, Bioorg. Chem. 2017;70:21021.

22. Md. Saifuzzaman, R. Morrison, Z. Zheng, S. Orive, J.Hamilton, P. E. Thompson , J. M. Al-rawi, Synthesis and biological evaluation of 8-aryl-2-morpholino-7-Osubstituted benzo[e][1,3]oxazin-4-ones against DNA-PK, PI3K, PDE3A enzymes and platelet aggregation. Bioorg. Med. Chem. 2017;25:5531-6.

23. Kaya O., Akçam, F.Z., Yayl. G., Investigation of the in vitro activities of various antibiotics against Brucella melitensis strains. Turk. J. Med. Sci., 2012;42:145-8.

24. Andrews J.M. Determination of minimum inhibitory concentrations. J. of Antimicrobial Chemotherapy 2001;48:5-16.

25. Krishnaiah D, Sarbatly R, Nithyanandam R. A review of the antioxidant potential of medicinal plant species. Food and Bioproducts Processing. 2011;89:217-33
26. Prior RL, Wu X, Schaich K. Standardized methods for the determination of antioxidant capacity and phenolics in foods and dietary supplements. J. of Agric. and Food Chem. 2005;53:4290-302.

27. Gil MI, Tomás-Barberán FA, Hess-Pierce B, Holcroft DM, Kader AA. Antioxidant activity of pomegranate juice and its relationship with phenolic composition and processing. J. of Agric. and Food Chem. 2000;48:4581-9

28. Chen Z, Bertin R, Froldi G., EC50 estimation of antioxidant activity in DPPH assay using several statistical programs. Food Chem. 2013;138:414-20

29. Gouhar RS, Abou-Elmagd WS, El-Zahar MI, Kamel MM, El-Ghonamy $\mathrm{DH}$. Synthesis of novel 5,6,7,8,9,10-hexahydropyrimido[4,5-b] quinoline derivatives for antimicrobial and anti-oxidant evaluation. Res Chem Intermed. 2017;43:1301-27.

30. Habib OM, Hassan HM, El-Mekabaty A. Studies on Some Benzoxazine-4-one Derivatives with Potential Biological Activity. American J. of Org. Chem. 2012;2:45-51

\section{GRAPHICAL ABSTRACT}
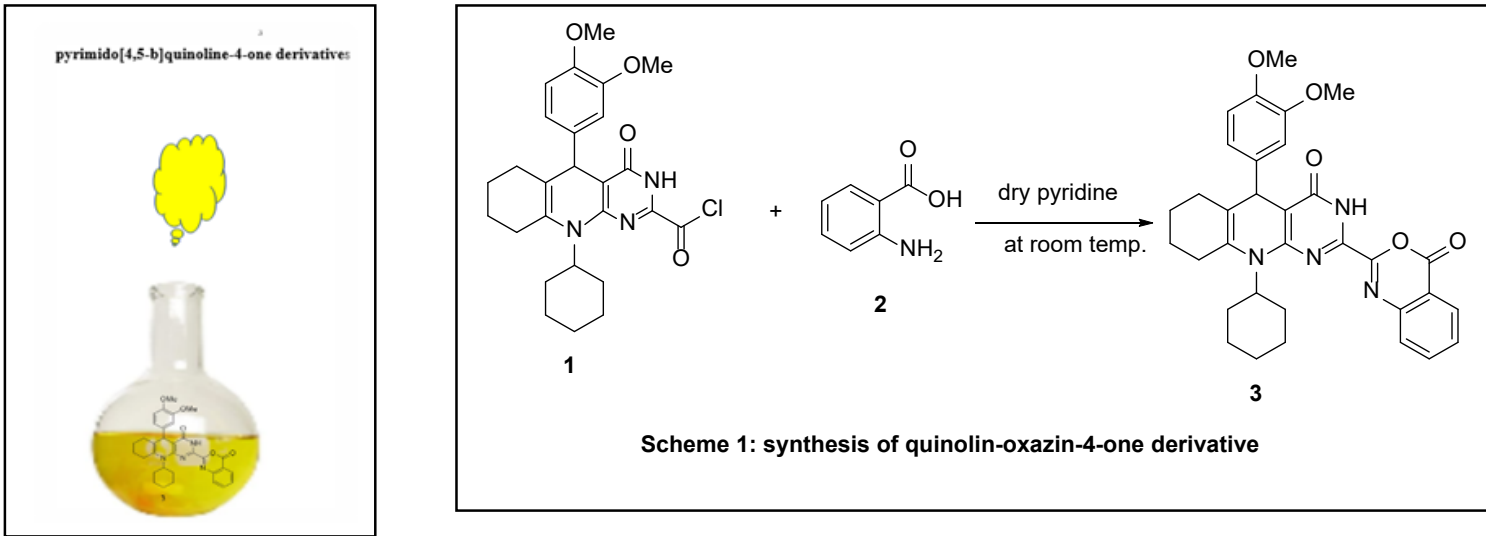

Scheme 1: synthesis of quinolin-oxazin-4-one derivative

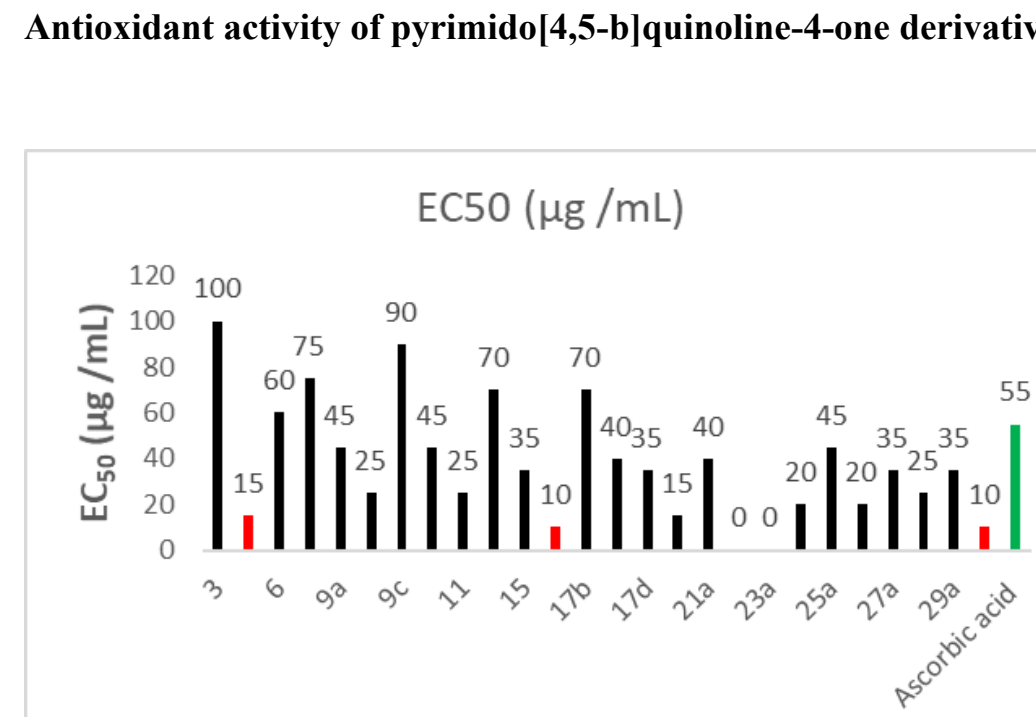

Compounds 


\section{ABOUT AUTHORS}

- Usama Fathy: Ass. Professor, Applied Organic Chemistry Department, National Research Centre, 33 El Bohouth st. (former EL Tahrir st.)-Dokki-Giza-Egypt-P.O.12622.

- Rasha S. Gouhar: Professor, Therapeutic Chemistry Department, National Research Centre, 33 El Bohouth st. (former EL Tahrir st.)-Dokki-Giza-Egypt-P.O.12622.

- Ahmed Younis: Ass. Professor, Green Chemistry Department, National Research Centre, 33 El Bohouth st. (former EL Tahrir st.)-Dokki-Giza-Egypt-P.O.12622.

- Dina H. El-Ghonemy: Ass. Professor, Microbial Chemistry Department, Genetic Engineering and Biotechnology Division, National Research Centre, 33 El Buhouth St., Dokki, Cairo, Egypt, P. C. 12622.

Cite this article: Fathy U, Gouhar RS, Younis A, El-Ghonemy DH. Synthesis of Novel pyrimido[4,5-b]quinoline-4-one Derivatives and Assessment as Antimicrobial and Antioxidant Agents. Pharmacog J. 2021;13(2): 550-62. 